\title{
THE DYNAMICAL 3-DIMENSIONAL INVERSE PROBLEM FOR THE MAXWELL SYSTEM
}

\author{
M. N. DEMCHENKO
}

To the memory of my father, N. P. Demchenko

\begin{abstract}
The problem of recovering the scalar electric permittivity and magnetic permeability (respectively, $\varepsilon$ and $\mu$ ) of a medium in a bounded domain $\Omega \subset \mathbb{R}^{3}$ by the boundary measurements on $\partial \Omega$ is considered. As data, the value of the velocity $c=(\varepsilon \mu)^{-1 / 2}$ with its normal derivative on $\partial \Omega$ is taken, along with the response operator, which maps the tangential part $\left.e_{\theta}\right|_{\partial \Omega \times[0,2 T]}$ of the electric field on the boundary to the tangential part $\left.h_{\theta}\right|_{\partial \Omega \times[0,2 T]}$ of the magnetic field $(2 T$ is the duration of measurements). With the help of the BC-method, it is established that the described data uniquely determine $\varepsilon$ and $\mu$ in the near-boundary layer with optical thickness $T$, provided that the domain satisfies some geometric condition.
\end{abstract}

\section{$\S 0$. INTRODUCTION}

Let $e, h$ be vector fields in a bounded domain $\Omega \subset \mathbb{R}^{3}, \partial \Omega \in C^{\infty}$, depending on time $t \in[0, T], T>0$, and let $f$ be a tangential field in $\partial \Omega$, also depending on $t$. The solution $\{e, h\}$ of the initial boundary-value problem

$$
\begin{aligned}
& e_{t}=\varepsilon^{-1} \operatorname{curl} h, \quad h_{t}=-\mu^{-1} \operatorname{curl} e, \quad(x, t) \in \Omega \times(0, T), \\
& \left.e\right|_{t=0}=\left.h\right|_{t=0}=0, \\
& \left.e_{\theta}\right|_{\partial \Omega \times[0, T]}=f
\end{aligned}
$$

in the space-time cylinder $\Omega \times[0, T]$ (here $(\cdot)_{t}$ means the derivative with respect to time $t,(\cdot)_{\theta}$ is the tangential part of the field on the boundary) describes the electromagnetic field induced by the boundary control $f$ in the medium with electric permittivity $\varepsilon$ and magnetic permeability $\mu$. We suppose that $\varepsilon$ and $\mu$ are smooth positive scalar functions on $\bar{\Omega}$. The inverse problem consists in recovering $\varepsilon$ and $\mu$ by the response operator $R^{T}: f \mapsto-\nu \times\left. h_{\theta}\right|_{\partial \Omega \times[0, T]}$, which describes the response of the system to different controls ( $\nu$ is the inward normal on the boundary, $\times$ is the vector product in $\mathbb{R}^{3}$ ). Since the electromagnetic waves propagate with the finite velocity

$$
c=(\varepsilon \mu)^{-1 / 2},
$$

we may find coefficients only in some subdomain $\Omega$ depending on $T$. Simple kinematic arguments show that for recovering the coefficients in the near-boundary layer $\Omega^{T}$ of optical thickness $T$ we need the response operator $R^{2 T}$. This is the time-optimal setup of the inverse problem. Note that we include the value of $c$ and of the normal derivative $\partial c / \partial \nu$ on $\partial \Omega$ in the data of the inverse problem (see Theorem 2).

In the papers [1, 2], where the inverse problem in the same setup was considered, the BC-method was used. This approach is based on the relationship between inverse

2010 Mathematics Subject Classification. Primary 35R30.

Key words and phrases. Inverse problem, Maxwell system, BC-method.

Supported by RFBR (grant no. 11-01-00407-a). 
problems and control theory (its description may be found in [3, 4]). In [2, it was proved that the velocity $c$ in $\Omega^{T}$ is uniquely determined by the data (2.5), provided that the layer $\Omega^{T}$ can be covered by semigeodesic coordinates with base on the boundary $\partial \Omega$; the problem of recovering $\varepsilon$ and $\mu$ separately was not considered. The paper 1 was devoted to the inverse problem for the Maxwell system on a 3-dimensional Riemannian manifold with boundary in the case where $\varepsilon=\mu=1$. The goal was to recover (up to isometry) the Riemannian manifold itself, or more precisely, to recover the near-boundary layer of thickness $T$ satisfying the same condition as in [2]. We also mention the papers [5, 6, 7, where the inverse problem for the Maxwell system was considered in a different (not time-optimal) setting.

In this paper, we generalize the result of 2 to the case of arbitrary $T$ and establish the uniqueness of $\varepsilon$ and $\mu$ in $\Omega^{T}$. We impose a geometric condition on the domain $\Omega^{T}$ and function $c$ (see Condition 1), besides the usual smoothness conditions. Before proving the uniqueness of $\varepsilon$ and $\mu$, we prove this for $c$, following the lines of [1, 2]. The main difficulty of this step is to adapt some constructions used in [1, 2] to the case where $T$ is arbitrary. Especially, this concerns the operator $M^{T}$ (see 4 ), which is the subject of the separate paper $[8]$.

Now we briefly describe the sections. In $\$ 1$ we define the necessary geometric notions. $\$ 2$ contains a description of some properties of problem (0.1), as well as of spaces and operators related to it, and the main result (Theorem 2). In \$3 we describe a model of the Maxwell system that can be obtained by the data of the inverse problem. \$4 is devoted to visualization, i.e., representation of the electric (magnetic) fields as tangential fields on $\partial \Omega \times[0, T]$, which we call images. In $\$ 5$ we give an amplitude formula, which is a necessary tool to obtain an image of a field induced by a given control, by using the data of the inverse problem. In $\$ 6$ we use these constructions to determine the velocity. Thus, we prove that the velocity $c$ in $\Omega^{T}$ is determined uniquely by the data of the inverse problem. In $\S \$ 79$ we prove the uniqueness of $\varepsilon$ and $\mu$. In $\$ 7$ we describe some constructions borrowed from [4]. \$8] contains some material of a technical nature. Finally, in 99 , we finish the proof of Theorem 2 .

The author thanks M. I. Belishev for posing an interesting problem and for attention to the author's work, and A. I. Karol for his advice concerning pseudodifferential operators and for other helpful discussions.

\section{§1. Geometry}

1.1. Optical metric, eikonal. Let $\Omega \subset \mathbb{R}^{3}$ be a bounded domain with boundary $\Gamma:=$ $\partial \Omega \in C^{\infty}$. We shall always assume that $\Gamma$ is connected. We introduce the velocity $c \in C^{\infty}(\bar{\Omega}), c>0$, which determines the optical metric in $\Omega$ :

$$
d s^{2}:=\frac{|d x|^{2}}{c^{2}}
$$

( $|d x|$ is Euclidean distance). The optical metric tensor $h$ is related to the Euclidean metric tensor as follows:

$$
h_{m n}=\frac{1}{c^{2}} g_{m n}, \quad h^{m n}=c^{2} g^{m n}, \quad m, n=1,2,3
$$

(these are relations for the covariant and contravariant tensors). Let $\operatorname{dist}_{c}(\cdot, \cdot)$ denote the distance function in the optical metric. We define the eikonal $\tau: \bar{\Omega} \rightarrow \overline{\mathbb{R}}_{+}$by the formula

$$
\tau(x):=\operatorname{dist}_{c}(x, \Gamma)
$$


We have $\tau \in \operatorname{Lip}(\bar{\Omega})$ because $\tau$ is the distance to a set. The eikonal satisfies the equation

$$
|\nabla \tau|=\frac{1}{c}
$$

Here and in the sequel, $\nabla$, curl, and div denote differential operators in the Euclidean metric.

We put

$$
T_{*}:=\max _{x \in \bar{\Omega}} \tau(x)
$$

Introduce the subdomains of $\Omega$ :

$$
\Omega^{s}:=\{x \in \Omega \mid \tau(x)<s\}
$$

and equidistant surfaces

$$
\Gamma^{s}:=\{x \in \Omega \mid \tau(x)=s\}
$$

where $s>0$. The field

is defined almost everywhere in $\bar{\Omega}$.

$$
\nu:=c \nabla \tau
$$

1.2. Semigeodesic coordinates, pattern. We denote by $l_{\gamma}$ a geodesic (with respect to the optical metric) that emanates from $\gamma \in \Gamma$ normally to the boundary, and by $l_{\gamma}[0, \tau]$ the segment of $l_{\gamma}$ of length $\tau>0\left(\tau\right.$ does not exceed the length of $\left.l_{\gamma}\right)$ that has one end at $\gamma$. The other end of $l_{\gamma}[0, \tau]$ is denoted by $x(\gamma, \tau)$. For every $\gamma \in \Gamma$, there exists a critical value $\tau_{*}(\gamma)$ such that for every $\tau<\tau_{*}(\gamma)$ the point $\gamma$ is a unique point of the boundary nearest to $x(\gamma, \tau)$, while for $\tau>\tau_{*}(\gamma)$ this is not true. The function $\tau_{*}$ is continuous on $\Gamma$. The separation set of the domain $\Omega$ with respect to the boundary $\Gamma$ is defined as follows:

$$
\omega:=\bigcup_{\gamma \in \Gamma} x\left(\gamma, \tau_{*}(\gamma)\right) .
$$

The set $\omega$ is closed and has zero Lebesgue measure.

Note that the function $\tau$ and the field $\nu$ are smooth outside of $\omega$ (here and in what follows, smoothness means $C^{\infty}$-smoothness). The surface $\Gamma^{s} \backslash \omega$ is also smooth, being a subset of a level set of function $\tau$. At the same time, for $x \in \Omega \backslash \omega$, the vector $\nu(x)$ is a unit vector normal to $\Gamma^{\tau(x)}$ at $x$, and it looks outward with respect to $\Omega^{\tau(x)}$.

If $x \in \Omega \backslash \omega$, then there exists a unique point of the boundary $\gamma(x)$ nearest to $x$. The map

$$
i: x \mapsto(\gamma(x), \tau(x))
$$

is a smooth diffeomorphism that maps $\Omega \backslash \omega$ to the set

$$
\Theta:=\left\{(\gamma, \tau) \mid \gamma \in \Gamma, 0<\tau<\tau_{*}(\gamma)\right\} \subset \Gamma \times \mathbb{R}_{+} .
$$

This set is called a pattern of the domain $\Omega$. For arbitrary local coordinates $\left(\gamma^{1}(\gamma), \gamma^{2}(\gamma)\right)$ on $\Gamma$, the collection $\left(\gamma^{1}(\gamma(x)), \gamma^{2}(\gamma(x)), \tau(x)\right)$ is called the semigeodesic coordinates (s.g.c.) of $x$. In these coordinates, the tensors $g$ and $h$ are written as

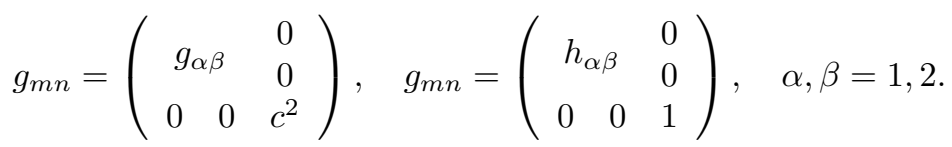

In what follows, we fix some number $T \in\left(0, T_{*}\right)$. The subset

$$
\Theta^{T}:=\left\{(\gamma, \tau) \in \Theta \mid \tau<\min \left(\tau_{*}(\gamma), T\right)\right\}
$$

of the pattern coincides with $i\left(\Omega^{T} \backslash \omega\right)$.

We say that a bounded domain $V \subset \mathbb{R}^{3}$ has Lipschitz boundary and write $\partial V \in$ Lip if in some neighborhood of any point on the boundary $\partial V$ the domain is a subgraph of a 
Lipschitz function. Now we can formulate assumptions concerning $\Omega$, the optical metric, and the number $T$, under which we solve the inverse problem.

Condition 1. The positive number $T$ satisfies the inequality $T<T_{*}$. For almost all $s \in(0, T)$, we have

$$
\partial \Omega^{s} \in \operatorname{Lip}, \quad \operatorname{meas}_{\Gamma^{s}}\left(\Gamma^{s} \cap \omega\right)=0,
$$

where $\operatorname{meas}_{\Gamma^{s}}$ is the surface measure on $\Gamma^{s}$. Moreover, (1.4) is true for $s=T$.

The cross-section of the pattern at the level $s>0$ provides a partition of $\Gamma$ into three subsets:

$$
\sigma_{+}^{s}:=\left\{\gamma \in \Gamma \mid \tau_{*}(\gamma)>s\right\}, \quad \sigma_{-}^{s}:=\left\{\gamma \in \Gamma \mid \tau_{*}(\gamma)<s\right\}, \quad \sigma_{\omega}^{s}:=\left\{\gamma \in \Gamma \mid \tau_{*}(\gamma)=s\right\} .
$$

Due to the continuity of $\tau_{*}$, the sets $\sigma_{+}^{s}$ and $\sigma_{-}^{s}$ are open. For different levels $s$, the sets $\sigma_{\omega}^{s}$ do not intersect, so that the set of numbers $s$ such that $\sigma_{\omega}^{s}$ has positive surface measure is finite or countable.

In what follows we shall use the same notation for scalar functions on $\Omega \backslash \omega$ and for their pullbacks to $\Theta$ by $i^{-1}$.

Our procedure of solving the inverse problem involves the following step: we recover the velocity $c$ in $\Omega^{T}$ by using the optical metric tensor in $\Theta^{T}$, which is pulled back by $i^{-1}$. In [2], the following theorem was proved.

Theorem 1. The tensor $h$ in $\Theta^{T}$ and the values of $c$ and $\partial c / \partial \nu$ on $\Gamma$ determine the velocity $\mathrm{c}$ in $\Omega^{T}$ uniquely.

1.3. Vector operations. The parentheses $\langle\cdot, \cdot\rangle$ denote the inner product in $\mathbb{R}^{3} ; \times$ is the vector product. We define operators acting on vector fields in $\bar{\Omega}$ :

$$
N z:=\nu \times z, \quad \Pi:=-N^{2} .
$$

The latter operator acts on fields as the pointwise orthogonal projection onto the plane tangential to $\Gamma^{\tau(x)}$ at $x$. In some cases we write $u_{\theta}$ instead of $\Pi u$.

By $\nabla_{\theta}$ we denote the operation $\Pi \nabla$. The same notation will be used in the following situation. Suppose a smooth function $\varphi$ is defined in $\Gamma^{s} \backslash \omega$. For a fixed point $x \in \Gamma^{s} \backslash \omega$, we choose some set $D \subset \Gamma^{s} \backslash \omega$ that is open in the relative topology of $\Gamma^{s}$ and contains $x$. Let $\tilde{\varphi}$ be some smooth extension of $\left.\varphi\right|_{D}$ to a neighborhood of $D$. We define the surface gradient of $\varphi$ at $x$ by the relation

$$
\left(\nabla_{\theta} \varphi\right)(x):=(\Pi \nabla \tilde{\varphi})(x) .
$$

Clearly this definition is consistent (it depends neither on the neighborhood of $D$, nor on the extension $\tilde{\varphi}$ ).

The adjoint operator to the minus surface gradient is the surface divergence of tangential fields in $\Gamma^{s} \backslash \omega$, to be denoted by $\operatorname{div}_{\theta}$.

\section{$\S 2$. The Dynamical Maxwell system}

2.1. Function spaces. Everywhere in this paper except $\$ 10$, we deal with real function spaces.

Denote by $\overrightarrow{\mathcal{L}}_{2}(\Gamma)$ the space of square integrable tangent fields on $\Gamma$. We introduce the space of controls

$$
\mathcal{F}^{T}:=L_{2}\left([0, T] ; \overrightarrow{\mathcal{L}}_{2}(\Gamma)\right)
$$

with the usual inner product

$$
\left(f, f^{\prime}\right)_{\mathcal{F}^{T}}:=\int_{\Gamma \times[0, T]}\left\langle f, f^{\prime}\right\rangle d \Gamma d t
$$


and the set $\mathcal{F}_{0}^{T}$ of smooth controls vanishing near $\Gamma \times\{t=0\}$. In terms of control theory, $\mathcal{F}^{T}$ is the exterior space of system (0.1).

We denote by $\vec{L}_{2, \eta}\left(\Omega^{T}\right)$ the space of fields in $\Omega^{T}$ that are square integrable with smooth positive weight $\eta$. We shall consider states $\{e(\cdot, t), h(\cdot, t)\}$ of the system as elements of the Hilbert space $J_{\varepsilon}^{T} \oplus J_{\mu}^{T}$, where the family of subspaces $J_{\eta}^{s} \subset \vec{L}_{2, \eta}\left(\Omega^{T}\right), s \in(0, T]$, is defined as follows:

$$
J_{\eta}^{s}:=\operatorname{clos}_{\vec{L}_{2, \eta}}\left\{y \in \vec{C}^{\infty}(\bar{\Omega}) \mid \operatorname{div}(\eta y)=0, \operatorname{supp} y \subset \Omega^{s} \cup \Gamma\right\} .
$$

The space $J_{\eta}^{s}$ consists of $\eta$-solenoidal fields having zero normal component on $\Gamma^{s}$ (in some generalized sense). This is true in the usual sense for fields $y \in \vec{C}^{\infty}\left(\overline{\Omega^{s}}\right) \cap J_{\eta}^{s}$ :

$$
\left.\langle y, \nu\rangle\right|_{\Gamma^{s} \backslash \omega}=0 .
$$

The sum $J_{\varepsilon}^{T} \oplus J_{\mu}^{T}$ is the interior space of the system (0.1). We introduce yet another family of subspaces of $\vec{L}_{2, \eta}\left(\Omega^{T}\right)$ :

$$
\mathcal{U}_{\eta}^{s}:=\cos _{\vec{L}_{2, \eta}}\left\{\eta^{-1} \operatorname{curl} z \mid z \in \vec{C}^{\infty}(\bar{\Omega}), \operatorname{supp} z \subset \Omega^{s} \cup \Gamma\right\} .
$$

Generally speaking, the space $\mathcal{U}_{\eta}^{s}$ does not coincide with (but is always a subspace of) $J_{\eta}^{s}$. The spaces $\mathcal{U}_{\eta}^{s}$ and $J_{\eta}^{s}$ may or may not coincide, depending on the topology of $\Omega^{s}$. The space $J_{\eta}^{s} \ominus \mathcal{U}_{\eta}^{s}$ consists of harmonic (with weight $\eta$ ) fields $\alpha$ satisfying two boundary conditions:

$$
\operatorname{div}(\eta \alpha)=0, \quad \operatorname{curl} \alpha=0,\left.\quad \alpha_{\theta}\right|_{\Gamma}=0,\left.\quad\langle\alpha, \nu\rangle\right|_{\Gamma^{s} \backslash \omega}=0 .
$$

Note that $\alpha \in \vec{C}^{\infty}\left(\overline{\Omega^{s} \backslash V}\right)$ for any neighborhood $V$ of the set $\Gamma^{s} \cap \omega$, so that the boundary conditions stated above may be treated in the usual sense. In [2] it was proved that if $s<\operatorname{dist}_{c}(\omega, \Gamma)$, then $J_{\eta}^{s}=\mathcal{U}_{\eta}^{s}$. This is not true for arbitrary $s$. However, the results of $\S 8.5$ in [9] show that if $\partial \Omega^{s} \in$ Lip, then

$$
\operatorname{dim} J_{\eta}^{s} \ominus \mathcal{U}_{\eta}^{s}<\infty
$$

Thus, (2.3) is true for all $s$ satisfying (1.4).

The use of the spaces $\mathcal{U}_{\eta}^{s}$ is motivated by the fact that for $f \in \mathcal{F}_{0}^{T}$ (in this case system (0.1) has a classical solution) we have

$$
\{e(\cdot, t), h(\cdot, t)\} \in \mathcal{U}_{\varepsilon}^{t} \oplus \mathcal{U}_{\mu}^{t}, \quad t \in(0, T] .
$$

Indeed, since the waves described by (0.1) propagate with finite velocity $c$, any boundary control $f$ acting during time period $t$ induces a field supported in a layer of optical thickness $t$, more precisely, in $\Omega^{t} \cup \Gamma$. We also need to check the identities

$$
e(\cdot, t)=\varepsilon^{-1} \operatorname{curl} z, \quad h(\cdot, t)=\mu^{-1} \operatorname{curl} z^{\prime}
$$

for $z, z^{\prime}$ with support in the same layer. This can be done by integrating the Maxwell equations over time.

2.2. Inverse problem. We introduce the response operator

$$
R^{T}: f \mapsto-\nu \times\left. h\right|_{\Gamma \times[0, T]},
$$

which acts in $\mathcal{F}^{T}$ and is well defined on $\mathcal{F}_{0}^{T}$. Also, we define the extended response operator denoted by $R^{2 T}$. For this, consider the following initial boundary-value problem:

$$
\begin{array}{ll}
e_{t}=\varepsilon^{-1} \operatorname{curl} h, & h_{t}=-\mu^{-1} \operatorname{curl} e, \quad x \in \Omega^{T}, \quad 0<t<2 T-\tau(x), \\
e=h=0, & x \in \Omega^{T}, \quad 0<t<\tau(x), \\
\left.e_{\theta}\right|_{\Gamma \times[0,2 T]}=f . &
\end{array}
$$


The operator $R^{2 T}$ acts in the space $\mathcal{F}^{2 T}$ by the rule

$$
R^{2 T}: f \mapsto-\nu \times\left. h\right|_{\Gamma \times[0,2 T]},
$$

which is similar to (2.4). The operator $R^{2 T}$ is well defined on $\mathcal{F}_{0}^{2 T}$. It can be shown that this operator has closure, which will be denoted by the same symbol. Obviously, $R^{2 T}$ is completely determined by the coefficients $\varepsilon, \mu$ in $\Omega^{T}$.

The extended response operator is treated as data in the inverse problem for the Maxwell system; it is used to recover $\varepsilon, \mu$ in $\Omega^{T}$. The following theorem is the main result of this paper.

Theorem 2. Suppose that the domain $\Omega$, the velocity $c$, and the number $T>0$ satisfy Condition 1. Then the data

$$
\left\{R^{2 T},\left.c\right|_{\Gamma},\left.\frac{\partial c}{\partial \nu}\right|_{\Gamma}\right\}
$$

determine functions $\varepsilon$ and $\mu$ in $\Omega^{T}$ uniquely.

In what follows we need the following fact proved in [2]: the data (2.5) determines $\varepsilon$ and $\mu$ on the boundary $\Gamma$.

2.3. Approximate controllability. With system (0.1), we associate the control operator $W^{T}: f \mapsto e(\cdot, T)$ acting from the control space $\mathcal{F}^{T}$ to the space $\mathcal{U}_{\varepsilon}^{T}$. This operator is well defined for $f \in \mathcal{F}_{0}^{T}$. The control operator is unbounded (see [2]) and has closure, which will still be denoted by $W^{T}$. Consider the space

$$
\mathcal{F}_{+}^{T}:=L_{2}\left([0, T] ; \vec{H}^{1 / 2}(\Gamma)\right),
$$

where $\vec{H}^{1 / 2}(\Gamma) \subset \overrightarrow{\mathcal{L}}_{2}(\Gamma)$ is the Sobolev vector space $W_{2}^{1 / 2}$. The map

$$
W^{T}: \mathcal{F}_{+}^{T} \rightarrow \mathcal{U}_{\varepsilon}^{T}
$$

is continuous (see [10).

The following fact was established in [2]:

$$
\cos _{J_{\varepsilon}^{T}} \operatorname{Ran} W^{T}=\mathcal{U}_{\varepsilon}^{T} .
$$

Relation (2.7) can be treated as approximate controllability of the Maxwell system. The proof of (2.7) is based on a vector version of the Holmgren-John-Tataru uniqueness theorem (see [11]). Approximate controllability plays an important role in the dynamical version of the BC-method.

Relation (2.7) can be written in another form. Define a set of smooth controls that lag behind by time $T-s(0<s<T)$ :

$$
\mathcal{F}_{0}^{T, s}:=\left\{f \in \mathcal{F}_{0}^{T} \mid \operatorname{supp} f \subset \Gamma \times(T-s, T]\right\} .
$$

The controls in $\mathcal{F}_{0}^{T, s}$ act during time $s$; therefore,

$$
\operatorname{clos}_{J_{\varepsilon}^{T}} W^{T} \mathcal{F}_{0}^{T, s}=\operatorname{clos}_{J_{\varepsilon}^{T}} \operatorname{Ran} W^{s} .
$$

If we change $T$ to $s$ in (2.7), then the last relation implies

$$
\operatorname{clos}_{J_{\varepsilon}^{T}} W^{T} \mathcal{F}_{0}^{T, s}=\mathcal{U}_{\varepsilon}^{s} .
$$


2.4. The connecting form. We define the connecting form $c^{T}$ on the set Dom $W^{T}$ :

$$
c^{T}\left[f, f^{\prime}\right]:=\left(W^{T} f, W^{T} f^{\prime}\right)_{J_{\varepsilon}^{T}}, \quad f, f^{\prime} \in \operatorname{Dom} W^{T} .
$$

The following result, obtained in [2], shows the relationship between the bilinear form $c^{T}$ and the response operator $R^{2 T}$. To formulate it, we need the operator of odd extension of controls from the interval $[0, T]$ to the interval $[0,2 T]$ :

$$
\left(S^{T} f\right)(\cdot, t):= \begin{cases}f(\cdot, t) & \text { if } 0 \leq t<T, \\ -f(\cdot, 2 T-t) & \text { if } T \leq t \leq 2 T,\end{cases}
$$

and we also need yet another space of controls

$$
\tilde{\mathcal{F}}_{0}^{T}:=\left\{f \in \mathcal{F}_{0}^{T} \mid S^{T} f \in \mathcal{F}_{0}^{2 T}\right\} .
$$

Proposition 1. For $f \in \widetilde{\mathcal{F}}_{0}^{T}$ and $f^{\prime} \in \mathcal{F}_{0}^{T}$, we have

$$
c^{T}\left[f, f^{\prime}\right]=\frac{1}{2}\left(\left(S^{T}\right)^{*} R^{2 T} S^{T} f, f^{\prime}\right)_{\mathcal{F}^{T}} .
$$

Identities of this type were first obtained by A. S. Blagoveshchenskiŭ.

Lemma 1. The operator $\left|W^{T}\right|=\left(\left(W^{T}\right)^{*} W^{T}\right)^{1 / 2}$ is uniquely determined by $R^{2 T}$.

Proof. Relation (2.9) shows that $R^{2 T}$ determines the form $c^{T}$ on $\tilde{\mathcal{F}}_{0}^{T}$ uniquely. The closure of this form has the same domain as the closure of $W^{T}$ defined initially on the set of controls $\tilde{\mathcal{F}}_{0}^{T}$. But $W^{T}$ acts continuously from $\mathcal{F}_{+}^{T}$ to $\mathcal{U}_{\varepsilon}^{T}$, and each of the sets $\tilde{\mathcal{F}}_{0}^{T}$ and $\mathcal{F}_{0}^{T}$ is dense in $\mathcal{F}_{+}^{T}$, so that the closure of $W^{T}$ with $\widetilde{\mathcal{F}}_{0}^{T}$ as its initial domain coincides with $W^{T}$. Therefore, the closure of $c^{T}$ from the set $\tilde{\mathcal{F}}_{0}^{T}$ is a symmetric bilinear form on Dom $W^{T}$, and the positive operator corresponding to it is $\left(W^{T}\right)^{*} W^{T}$. Thus, starting with $R^{2 T}$, we can recover the operator $\left|W^{T}\right|=\left(\left(W^{T}\right)^{*} W^{T}\right)^{1 / 2}$ acting in the space $\mathcal{F}^{T}$.

2.5. The Maxwell system with magnetic control. Everything that was said above about system (0.1) holds true for the Maxwell system with magnetic control $g$ :

$$
\begin{aligned}
& e_{t}=\varepsilon^{-1} \operatorname{curl} h, \quad h_{t}=-\mu^{-1} \operatorname{curl} e, \quad(x, t) \in \Omega \times(0, T), \\
& \left.e\right|_{t=0}=\left.h\right|_{t=0}=0, \\
& \left.h_{\theta}\right|_{\Gamma \times[0, T]}=g .
\end{aligned}
$$

With this system, we associate the control operator $W_{m}^{T}: g \mapsto h(\cdot, T) \in \mathcal{U}_{\mu}^{T}$ and the response operator $R_{m}^{T}: g \mapsto \nu \times\left. e_{\theta}\right|_{\Gamma \times[0, T]}$. We need a relationship between $R_{m}^{T}$ and $R^{T}$. First, we observe the obvious inclusions

$$
R^{T} \mathcal{F}_{0}^{T}, R_{m}^{T} \mathcal{F}_{0}^{T} \subset \mathcal{F}_{0}^{T} .
$$

Now, suppose that $\{e, h\}$ solves problem (0.1) with control $f \in \mathcal{F}_{0}^{T}$. By (2.4), this solution coincides with the solution (2.10) for the control $g=N R^{T} f$ (the operator $N$ acts on fields in $\mathcal{F}^{T}$ by the rule similar to (1.5)); therefore, $R_{m}^{T} N R^{T} f=\left.N e\right|_{\Gamma \times[0, T]}=N f$, or

$$
-N R_{m}^{T} N R^{T} f=f .
$$

If we interchange systems (0.1) and (2.10) in this argument, we obtain

$$
N R^{T}\left(-N R_{m}^{T}\right) g=g .
$$

Thus, the operators $N R^{T}$ and $-N R_{m}^{T}$ are mutually inverse, and

$$
R_{m}^{T}=-N\left(R^{T}\right)^{-1} N
$$


This simple relation allows us to pass from $R^{2 T}$, which is contained in the data of the inverse problem, to the operator $R_{m}^{2 T}$. Using the latter we obtain the operator $\left|W_{m}^{T}\right|$ by the "magnetic" connecting form

$$
c_{m}^{T}\left[g, g^{\prime}\right]:=\left(W_{m}^{T} g, W_{m}^{T} g^{\prime}\right)_{J_{\mu}^{T}}, \quad g, g^{\prime} \in \operatorname{Dom} W_{m}^{T},
$$

because there is a relation similar to (2.9):

$$
c_{m}^{T}\left[g, g^{\prime}\right]=\frac{1}{2}\left(\left(S^{T}\right)^{*} R_{m}^{2 T} S^{T} g, g^{\prime}\right)_{\mathcal{F}^{T}} .
$$

The operators $\left|W^{T}\right|,\left|W_{m}^{T}\right|$ are used in a model of systems (0.1), (2.10). Now we turn to the description of this model.

\section{§3. The Model of the Dynamical Maxwell System}

3.1. Model spaces and operators. To solve the inverse problem, we pass from the interior spaces and operators to their model counterparts, which can be obtained from the data (2.5). The model spaces describing the states of the system are defined as follows:

$$
\mathcal{U}_{\varepsilon \#}^{T}:=\mathcal{U}_{\mu \#}^{T}:=\mathcal{F}^{T} .
$$

Next we introduce the operators $\Phi^{T}: \mathcal{U}_{\varepsilon \#}^{T} \rightarrow \mathcal{U}_{\varepsilon}^{T}$ and $\Phi_{m}^{T}: \mathcal{U}_{\mu \#}^{T} \rightarrow \mathcal{U}_{\mu}^{T}$ via the polar decompositions

$$
W^{T}=\Phi^{T}\left|W^{T}\right|, \quad W_{m}^{T}=\Phi_{m}^{T}\left|W_{m}^{T}\right| .
$$

In [2] it was proved that the kernel of $W^{T}$ is trivial for $T<T_{*}$. Thus,

$$
\operatorname{clos}_{\mathcal{F}^{T}} \operatorname{Ran}\left|W^{T}\right|=\left(\operatorname{Ker}\left|W^{T}\right|\right)^{\perp}=\left(\operatorname{Ker} W^{T}\right)^{\perp}=\mathcal{F}^{T} .
$$

Together with (2.7), this implies that the operator $\Phi^{T}$ is unitary. This is also true for $\Phi_{m}^{T}$.

We may say that the collection

$$
\left\{\mathcal{U}_{\varepsilon \#}^{T}, \mathcal{U}_{\mu \#}^{T},\left|W^{T}\right|,\left|W_{m}^{T}\right|\right\}
$$

forms a model of the dynamical system in question. Note that passage from the original system to its model is traditional in the BC-method.

Below, we shall need the following model spaces:

$$
\mathcal{U}_{\varepsilon \#}^{s}:=\left(\Phi^{T}\right)^{*} \mathcal{U}_{\varepsilon}^{s} \subset \mathcal{U}_{\varepsilon \#}^{T} .
$$

In the following calculation we use (2.8) and (3.1):

$$
\mathcal{U}_{\varepsilon \#}^{s}=\left(\Phi^{T}\right)^{*} \mathcal{U}_{\varepsilon}^{s}=\left(\Phi^{T}\right)^{*} \operatorname{clos}_{J_{\varepsilon}^{T}} W^{T} \mathcal{F}_{0}^{T, s}=\cos _{\mathcal{F}^{T}}\left|W^{T}\right| \mathcal{F}_{0}^{T, s} .
$$

It is important that, since the operator $\left|W^{T}\right|$ is determined by the data of the inverse problem, the last representation shows that the same data also determine the subspaces $\mathcal{U}_{\varepsilon \#}^{s}$. This is also true for the "magnetic" subspaces:

$$
\mathcal{U}_{\mu \#}^{s}:=\left(\Phi_{m}^{T}\right)^{*} \mathcal{U}_{\mu}^{s} \subset \mathcal{U}_{\mu \#}^{T} \cdot
$$

3.2. Model Maxwell operator. Next we obtain a unitary copy of the Maxwell operator within the model (3.2). We consider, for example, the block $\mu^{-1}$ curl acting from $\mathcal{U}_{\varepsilon}^{T}$ to $\mathcal{U}_{\mu}^{T}$.

We introduce the set of smooth controls vanishing at $t=T$,

$$
\mathcal{F}_{00}^{T}:=\left\{f \in \mathcal{F}_{0}^{T} \mid f(\cdot, T)=0\right\},
$$

and consider the following set of elements of the space $\mathcal{U}_{\varepsilon}^{T} \oplus \mathcal{U}_{\mu}^{T}$ :

$$
\left\{\left\{W^{T} f,-W_{m}^{T} N R^{T} f_{t}\right\} \mid f \in \mathcal{F}_{00}^{T}\right\} .
$$

This set forms a graph of some restriction of the maximal operator $\mu^{-1}$ curl. This can be shown as follows. Let $\{e, h\}$ be a (classical) solution of system (0.1) with control $f \in \mathcal{F}_{0}^{T}$. 
It coincides with the solution of system (2.10) for the control $g=N R^{T} f$, while $\left\{e_{t}, h_{t}\right\}$ is a solution of (2.10) with control $\left(N R^{T} f\right)_{t}=N R^{T} f_{t}$, whence

$$
-W_{m}^{T} N R^{T} f_{t}=-h_{t}(\cdot, T)=\mu^{-1} \operatorname{curl} e(\cdot, T)=\mu^{-1} \operatorname{curl} W^{T} f
$$

(in the second identity we have used the second equation in (0.1)). The closure of the set (3.4) in the norm of $\mathcal{U}_{\varepsilon}^{T} \oplus \mathcal{U}_{\mu}^{T}$ forms a graph of a closed linear operator. We denote this operator by $\mathcal{R}_{e}^{T}$.

Note that $\mathcal{R}_{e}^{T}$ is densely defined in $\mathcal{U}_{\varepsilon}^{T}$, because $\operatorname{Dom} \mathcal{R}_{e}^{T}$ contains $W^{T} \mathcal{F}_{00}^{T}$ by construction. The latter set is dense in $\mathcal{U}_{\varepsilon}^{T}$, which follows from the fact that $\mathcal{F}_{00}^{T}$ is dense in $\mathcal{F}_{+}^{T}$, the continuity of the map (2.6), and identity (2.7). However, we need a stronger fact concerning the domain of $\mathcal{R}_{e}^{T}$. Before we formulate it (Lemma 2) we give the following definition: we shall say that a field $y \in \vec{L}_{2}(U)\left(U\right.$ is some domain in $\left.\mathbb{R}^{3}\right)$ such that curl $y \in \vec{L}_{2}(U)$ satisfies the condition $\left.y_{\theta}\right|_{\partial U}=0$ if

$$
(\operatorname{curl} y, z)_{\vec{L}_{2}(U)}=(y, \operatorname{curl} z)_{\vec{L}_{2}(U)}
$$

for any $z \in \vec{L}_{2}(U)$ such that $\operatorname{curl} z \in \vec{L}_{2}(U)$.

For a smooth positive weight $\eta$ in $\overline{\Omega^{T}}$, we introduce the set $\mathcal{W}_{\eta}^{T} \subset \mathcal{U}_{\eta}^{T}$ that consists of all elements $y$ such that $y \in \mathcal{U}_{\eta}^{s}$ for some $s \in(0, T)$ depending on $y$ (we assume that $y$ is extended by zero to $\left.\Omega^{T} \backslash \Omega^{s}\right)$, and curl $y \in \vec{L}_{2}\left(\Omega^{T}\right),\left.y_{\theta}\right|_{\partial \Omega^{T}}=0$. Obviously, $\mathcal{W}_{\varepsilon}^{T}$ is wider than $W^{T} \mathcal{F}_{00}^{T}$.

Lemma 2. We have $\mathcal{W}_{\varepsilon}^{T} \subset \operatorname{Dom} \mathcal{R}_{e}^{T}$.

The proof of this lemma is given in the Appendix.

The graph (3.4) has an important feature: its unitary copy in $\mathcal{U}_{\varepsilon \#}^{T} \oplus \mathcal{U}_{\mu \#}^{T}$,

$$
\left\{\left\{\left|W^{T}\right| f,-\left|W_{m}^{T}\right| N R^{T} f_{t}\right\} \mid f \in \mathcal{F}_{00}^{T}\right\},
$$

can be obtained by using the data of the inverse problem. This copy is mapped to the set (3.4) under $\Phi^{T} \oplus \Phi_{m}^{T}$. The closure of the above subset in $\mathcal{U}_{\varepsilon \#}^{T} \oplus \mathcal{U}_{\mu \#}^{T}$ is a graph of a linear operator to be denoted by $\mathcal{R}_{e \#}^{T}$. This operator acts from $\mathcal{U}_{\varepsilon \#}^{T}$ to $\mathcal{U}_{\mu \#}^{T}$ and is unitarily equivalent to $\mathcal{R}_{e}^{T}$ :

$$
\mathcal{R}_{e \#}^{T}=\left(\Phi_{m}^{T}\right)^{*} \mathcal{R}_{e}^{T} \Phi^{T} .
$$

Within the inverse problem, it is essential that the operator $\mathcal{R}_{e \#}^{T}$, being determined by (3.4), be determined by the data (2.5). Below we shall use the operator $\mathcal{R}_{\text {e\# }}^{T}$ to find the parameters of the medium in $\Omega^{T}$. But before doing this, we need to describe a representation of waves as tangential fields in the pattern; such fields are called images.

\section{$\S 4$. Wave IMAGES}

Since the image of a field $y \in \mathcal{U}_{\eta}^{T}$ is constructed from discontinuities of the projections of $y$ to $\mathcal{U}_{\eta}^{s}$, we need a description of these projections.

4.1. The projections $P_{\eta}^{s}, E_{\eta}^{s}$, and $B_{\eta}^{s}$. The orthogonal projections onto $J_{\eta}^{s}, \mathcal{U}_{\eta}^{s}$, and $J_{\eta}^{s} \ominus \mathcal{U}_{\eta}^{s}$, acting in $J_{\eta}^{T}$, are denoted by $P_{\eta}^{s}, E_{\eta}^{s}$, and $B_{\eta}^{s}$, respectively.

We introduce the family of operators $\left\{\widetilde{Q}_{\eta}^{s}\right\}$ defined for a.e., $s \in(0, T]$ and acting by the following rule. Let $\psi$ be a bounded function on $\Gamma^{s}$ that is smooth on $\Gamma^{s} \backslash \omega$, and let $s$ satisfy (1.4). Then there exists a unique solution $p^{s} \in H^{1}\left(\Omega^{s}\right)$ of the following boundary-value problem in $\Omega^{s}$ :

$$
\operatorname{div}\left(\eta \nabla p^{s}\right)=0,\left.\quad p^{s}\right|_{\Gamma}=0,\left.\quad \frac{\partial p^{s}}{\partial \nu}\right|_{\Gamma^{s}}=\left.\psi\right|_{\Gamma^{s}} .
$$


By definition, the operator $\widetilde{Q}_{\eta}^{s}$ maps $\psi$ to $p^{s}$. Also, we introduce the operators $Q_{\eta}^{s}$ that act on a bounded function $\varphi$ in $\Omega^{T}$ smooth outside of $\omega$ by the rule

$$
Q_{\eta}^{s} \varphi:=\widetilde{Q}_{\eta}^{s}\left(\left.\varphi\right|_{\Gamma^{s}}\right)
$$

Since $\partial \Omega^{s} \in$ Lip, the Helmholtz decomposition in the domain $\Omega^{s}$ is possible (see, e.g., [12]):

$$
\vec{L}_{2, \eta}\left(\Omega^{s}\right)=\left\{\nabla \varphi\left|\varphi \in H^{1}\left(\Omega^{s}\right), \varphi\right|_{\Gamma}=0\right\} \oplus J_{\eta}^{s} .
$$

Then for $y \in \vec{C}^{\infty}\left(\overline{\Omega^{T}}\right) \cap J_{\eta}^{T}$ we have

$$
\left.\left(y-P_{\eta}^{s} y\right)\right|_{\Omega^{s}}=\nabla p^{s}, \quad p^{s} \in H^{1}\left(\Omega^{s}\right),\left.\quad p^{s}\right|_{\Gamma}=0 .
$$

Since the identity $y-\nabla p^{s}=P_{\eta}^{s} y$ is true in $\Omega^{s}$, we have

$$
\left(\nabla p^{s}, \nabla \varphi\right)_{\vec{L}_{2, \eta}\left(\Omega^{s}\right)}=(y, \nabla \varphi)_{\vec{L}_{2, \eta}\left(\Omega^{s}\right)}, \quad \varphi \in H^{1}\left(\Omega^{s}\right),\left.\quad \varphi\right|_{\Gamma}=0 .
$$

Integrating by parts on the right, we arrive at the boundary-value problem (4.1) for a function $p^{s}$ whose normal derivative on the boundary is $\psi=\langle y, \nu\rangle$. Thus, $p^{s}=Q_{\eta}^{s}\langle y, \nu\rangle$, and we can write (4.2) as follows:

$$
\left.P_{\eta}^{s} y\right|_{\Omega^{s}}=\left.y\right|_{\Omega^{s}}-\nabla Q_{\eta}^{s}\langle y, \nu\rangle .
$$

Of course, the projection $P_{\eta}^{s} y$ vanishes in $\Omega^{T} \backslash \Omega^{s}$. Since

$$
E_{\eta}^{s}=P_{\eta}^{s}-B_{\eta}^{s}
$$

we have

$$
\left.E_{\eta}^{s} y\right|_{\Omega^{s}}=\left.\left(y-\nabla Q_{\eta}^{s}\langle y, \nu\rangle-B_{\eta}^{s} y\right)\right|_{\Omega^{s}} .
$$

In Subsection 2.1 we noted that every field in $J_{\eta}^{s} \ominus \mathcal{U}_{\eta}^{s}$ (in particular, $B_{\eta}^{s} y$ ) is smooth in $\overline{\Omega^{s}}$ outside of an arbitrary neighborhood of $\Gamma^{s} \cap \omega$. The same can be said about $Q_{\eta}^{s}\langle y, \nu\rangle$, because it is a solution of the elliptic boundary-value problem (4.1) with $\psi=\langle y, \nu\rangle$, and the surface $\Gamma^{s} \backslash \omega$ is smooth, together with $\psi$ on this surface. Therefore, the restriction $E_{\eta}^{s} y$ to $\Omega^{s}$ has a trace on $\Gamma^{s}$, to be denoted by $\left.E_{\eta}^{s} y\right|_{\Gamma^{s-0}}$. This trace is smooth outside of $\Gamma^{s} \cap \omega$, and the following relation holds true:

$$
\left.E_{\eta}^{s} y\right|_{\Gamma^{s-0}}=\left.\left(y_{\theta}-\nabla_{\theta} Q_{\eta}^{s}\langle y, \nu\rangle-B_{\eta}^{s} y\right)\right|_{\Gamma^{s-0}} .
$$

Here we have used the previous identity and the Neumann boundary condition in (4.1). Recalling the last relation in (2.2) for $\alpha=B_{\eta}^{s} y$, we obtain

$$
\left.\left\langle E_{\eta}^{s} y, \nu\right\rangle\right|_{\Gamma^{s-0}}=0
$$

i.e., the trace $\left.E_{\eta}^{s} y\right|_{\Gamma^{s-0}}$ is a tangential field on $\Gamma^{s}$.

4.2. $M^{T}$-transformation. Here we define an operator

$$
M_{\eta}^{T}: \mathcal{U}_{\eta}^{T} \rightarrow \overrightarrow{\mathcal{L}}_{2, \eta}\left(\Omega^{T}\right),
$$

where $\eta$ is some smooth positive weight in $\overline{\Omega^{T}}$ and $\overrightarrow{\mathcal{L}}_{2, \eta}\left(\Omega^{T}\right)$ is the subspace of transversal fields in $\vec{L}_{2, \eta}\left(\Omega^{T}\right)$,

$$
\overrightarrow{\mathcal{L}}_{2, \eta}\left(\Omega^{T}\right):=\left\{v \in \vec{L}_{2, \eta}\left(\Omega^{T}\right) \mid\langle v(x), \nu(x)\rangle=0, x \in \Omega^{T}\right\} .
$$

We define the image $M_{\eta}^{T} y$ of $y \in \vec{C}^{\infty}\left(\overline{\Omega^{T}}\right) \cap \mathcal{U}_{\eta}^{T}$ by specifying its values on the equidistant surfaces $\Gamma^{s}$ :

$$
\left.M_{\eta}^{T} y\right|_{\Gamma^{s}}:=\left.E_{\eta}^{s} y\right|_{\Gamma^{s-0}}
$$


Like in (4.5), here it is assumed that $s$ satisfies (1.4), and (4.7) is valid on $\Gamma^{s} \backslash \omega$, hence, a.e. on $\Gamma^{s}$. Since (1.4) is true for a.e. $s$, formula (4.7) uniquely determines the field $M_{\eta}^{T} y$ a.e. in $\Omega^{T}$. Relation (4.6) shows that a.e. we have

$$
\left\langle M_{\eta}^{T} y, \nu\right\rangle=0 .
$$

The definition is consistent if the field $M_{\eta}^{T}$ is square integrable. This is obvious if $\overline{\Omega^{T}} \cap \omega=$ $\varnothing$, because this condition implies that of all equidistant surfaces $\Gamma^{s}$ are smooth, $s \in(0, T]$. Moreover, in [2, 13] it was proved that under this assumption $M_{\eta}^{T}$ is unitary. However, for arbitrary $T<T_{*}$ even the consistency of the above definition is nontrivial, because the equidistant surfaces $\Gamma^{s}$ where the Neumann boundary condition is posed in (4.1) may fail to be smooth and may depend nonregularly on the parameter $s$.

The case of an arbitrary $T$ was treated in the paper [8], where some formula for $M_{\eta}^{T}$ involving an operator integral was obtained. The main result of $[8]$ can be formulated as follows.

Theorem 3. a) If $y \in \vec{C}^{\infty}\left(\overline{\Omega^{T}}\right) \cap \mathcal{U}_{\eta}^{T}$, then $M_{\eta}^{T} y \in \overrightarrow{\mathcal{L}}_{2, \eta}\left(\Omega^{T}\right)$. The closure of the operator $M_{\eta}^{T}$, which will be denoted by the same symbol, is a bounded operator from $\mathcal{U}_{\eta}^{T}$ to $\overrightarrow{\mathcal{L}}_{2, \eta}\left(\Omega^{T}\right)$.

b) We have

$$
M_{\eta}^{T}\left(M_{\eta}^{T}\right)^{*}=\mathbb{1}
$$

(the right-hand side is the identity operator in $\overrightarrow{\mathcal{L}}_{2, \eta}\left(\Omega^{T}\right)$ ).

c) If $v \in \vec{C}_{0}^{\infty}\left(\Omega^{T} \backslash \omega\right) \cap \overrightarrow{\mathcal{L}}_{2, \eta}\left(\Omega^{T}\right)$, then

$$
\left(M_{\eta}^{T}\right)^{*} v \in \mathcal{W}_{\eta}^{T}
$$

Note that, in [8], $M_{\eta}^{T} y$ was defined on $\Gamma^{s}$ as $\left.P_{\eta}^{s} y\right|_{\Gamma^{s-0}}$. This differs from (4.7), but Theorem 3 can be proved in almost the same way.

Item (b) shows that the operator $M_{\eta}^{T}$ is partially isometric and its range coincides with $\overrightarrow{\mathcal{L}}_{2}\left(\Omega^{T}\right)$. However, its kernel may be nontrivial (and infinite-dimensional). The results of [8] imply that $\operatorname{Ker} M_{\eta}^{T}$ contains the singular subspace of the spectral family $\left\{E_{\eta}^{s}\right\}$, which may have jumps of infinite dimension. Whether or not $\left\{E_{\eta}^{s}\right\}$ can have a singular continuous component is an open question.

Observe the following intertwining property of $M_{\eta}^{T}$ :

$$
M_{\eta}^{T} E_{\eta}^{s}=X^{s} M_{\eta}^{T},
$$

where $X^{s}$ is the operator that multiplies a field by the characteristic function of $\Omega^{s}$.

4.3. Image operator. We define a subspace of fields in $\mathcal{F}^{T}$ supported on the pattern:

$$
\overrightarrow{\mathcal{L}}_{2}\left(\Theta^{T}\right):=\left\{f \in \mathcal{F}^{T} \mid \operatorname{supp} f \subset \overline{\Theta^{T}}\right\} .
$$

Let $\pi^{s}$ be the following pointwise transformation of tangential fields on $\Gamma^{s} \backslash \omega$ to tangential fields on the set $\sigma_{+}^{s}$ (see Subsection 1.2): $\left(\pi^{s} u\right)(\gamma)$ is the parallel transition (with respect to the optical metric) of the vector $u(x(\gamma, s))$ from $x(\gamma, s)$ to $\gamma$ along the geodesic $l_{\gamma}[0, s]$ connecting these points. Also, we define the operation $\pi$ that acts on transversal vector fields in $\Omega^{T}$ by the rule

$$
(\pi u)(\gamma, s):=\left(\pi^{s}\left(\left.u\right|_{\Gamma^{s}}\right)\right)(\gamma), \quad(\gamma, s) \in \Theta^{T}
$$

For $u \in \overrightarrow{\mathcal{L}}_{2}\left(\Omega^{T}\right)$, the image $\pi u$ belongs to $\overrightarrow{\mathcal{L}}_{2}\left(\Theta^{T}\right)$.

We set $c_{0}(x):=c(\gamma(x))$, where the right-hand side is the restriction of $c$ to $\Gamma$. We define a function $\varkappa$ in $\Omega \backslash \omega$ as follows. Let $(\gamma, \tau) \in \Theta$. For some fixed coordinates 
$\left(\gamma^{1}, \gamma^{2}, \tau\right)$, we set

$$
\varkappa:=\frac{c^{3 / 2}}{c_{0}}\left(\frac{\operatorname{det}\left\{g_{\alpha \beta}\left(\gamma^{1}, \gamma^{2}, \tau\right)\right\}}{\operatorname{det}\left\{g_{\alpha \beta}\left(\gamma^{1}, \gamma^{2}, 0\right)\right\}}\right)^{1 / 4} .
$$

It is easily seen that $\varkappa$ does not depend on choice of the local coordinates $\left(\gamma^{1}, \gamma^{2}\right)$. The ratio of determinants in this formula has the meaning of the square of the divergence of geodesics emanating normally from the boundary. We introduce yet another function on $\Omega \backslash \omega$ :

$$
\varkappa_{\eta}:=\varkappa \eta^{1 / 2}
$$

and finally define the image operator by

$$
I_{\eta}^{T}:=\pi \varkappa_{\eta} M_{\eta}^{T}
$$

It is convenient to view $I_{\eta}^{T}$ as an operator acting from $\mathcal{U}_{\eta}^{T}$ to $\mathcal{F}^{T}$ in accordance with the evident imbedding $\overrightarrow{\mathcal{L}}_{2}\left(\Theta^{T}\right)$ to $\mathcal{F}^{T}$. Direct calculations show that the composition $\pi \varkappa_{\eta}$ is a unitary operator from $\overrightarrow{\mathcal{L}}_{2}\left(\Omega^{T}\right)$ to $\overrightarrow{\mathcal{L}}_{2}\left(\Theta^{T}\right)$. Together with Theorem 3 , this means that $I_{\eta}^{T}$ is a partially isometric operator with

$$
\operatorname{Ran} I_{\eta}^{T}=\overrightarrow{\mathcal{L}}_{2}\left(\Theta^{T}\right),
$$

because $\operatorname{Ran} M_{\eta}^{T}=\overrightarrow{\mathcal{L}}_{2}\left(\Omega^{T}\right)$. Moreover, we have

$$
\left(I_{\eta}^{T}\right)^{*}=\left(M_{\eta}^{T}\right)^{*} \varkappa_{\eta}^{-1} \pi^{-1}
$$

(here $\pi^{-1}$ acts on the restriction of a field in $\mathcal{F}^{T}$ to the set $\Theta^{T}$ ).

In the case of a regular zone $\left(\Omega^{T} \cap \omega=\varnothing\right)$, the set $\Theta^{T}$ coincides with the cylinder $\Gamma \times(0, T)$, and the operator $I_{\eta}^{T}$ becomes unitary, which allows one to use the space $\mathcal{F}^{T}$ as a model space for system (0.1). This functional model was used in 1, 2, for recovering the optical metric in $\Omega^{T}$ and the velocity. In general, the operator $I_{\eta}^{T}$ may have nontrivial kernel (together with $M_{\eta}^{T}$ ), so that the image on the pattern is not a full description of a field. Nevertheless, images allow us to find the velocity $c$ in $\Omega^{T}$.

\section{$\S 5$. Amplitude formula}

In this section we show how to construct the image of a wave $W^{T} f$ for an arbitrary control $f \in \mathcal{F}_{0}^{T}$ by using only the data of the inverse problem (2.5) and the model (3.2). We borrow this technique from [2]. The key role is played by the amplitude formula (5.1), which can be obtained with the help of the ray method. This formula contains the operator $\left(W^{T}\right)^{*}$, which acts continuously from $\mathcal{U}_{\varepsilon}^{T}$ to the following space of controls:

$$
\mathcal{F}_{-}^{T}:=\left(\mathcal{F}_{+}^{T}\right)^{\prime}=L_{2}\left((0, T) ; \vec{H}^{-1 / 2}(\Gamma)\right) .
$$

This fact follows from the continuity of the map (2.6). The action of the operator $\left(W^{T}\right)^{*}$ on the field $y$ can be described in terms of the initial boundary-value problem for Maxwell equations, which is adjoint to problem (0.1) and contains $y$ as initial data (see details in the Appendix).

The amplitude formula describes the action of the operator $\left(W^{T}\right)^{*}$ on the field $\left(E_{\varepsilon}^{s}\right)^{\perp} y$, $s \in(0, T)$. Formulas (4.4) and (4.5) imply that on the surface $\Gamma^{s}$ the field $\left(E_{\varepsilon}^{s}\right)^{\perp} y=$ $y-E_{\varepsilon}^{s} y$ has a jump equal to $\left.E_{\varepsilon}^{s} y\right|_{\Gamma^{s-0}}$; therefore, the proof of the amplitude formula reduces to the analysis of the solution of the adjoint problem with discontinuous initial data. This analysis can be done with the help of the ray method, and we give only the result.

Lemma 3. Suppose that $s \in(0, T)$ satisfies (1.4), and that $y \in \mathcal{U}_{\varepsilon}^{T} \cap \vec{C}^{\infty}\left(\overline{\Omega^{T}}\right)$. Let $v:=\left(W^{T}\right)^{*}\left(E_{\varepsilon}^{s}\right)^{\perp} y \in \mathcal{F}_{-}^{T}$. Then: 
a) the restriction of $v$ to $\Gamma \times(T-s, T)$ vanishes;

b) for all $\gamma \in \sigma_{+}^{s}$, there exists an open neighborhood $D \subset \Gamma$ containing $\gamma$ and a number $\delta>0$ such that the restriction of $v$ to $D \times(T-s-\delta, T-s)$ is continuous, has a trace on $D \times\{T-s\}$, and satisfies

$$
\lim _{\tau \rightarrow T-s-0} v(\gamma, \tau)=\varkappa_{\varepsilon}(\gamma, 0) \varkappa_{\varepsilon}(\gamma, s)\left(\pi^{s}\left(\left.E_{\varepsilon}^{s} y\right|_{\Gamma^{s-0}}\right)\right)(\gamma)
$$

c) for any $\gamma \in \sigma_{-}^{s}$ there exists an open neighborhood $D \subset \Gamma$ containing $\gamma$ and a number $\delta>0$ such that the restriction of $v$ to $D \times(T-s-\delta, T-s)$ vanishes.

Formula (5.1) can be applied to $y=W^{T} f, f \in \mathcal{F}_{0}^{T}$, because in this case the field $y$ is smooth. Then on the left-hand side we have a limit of the field $v^{s}:=\left(W^{T}\right)^{*}\left(E_{\varepsilon}^{s}\right)^{\perp} W^{T} f$. The projection $E_{\varepsilon}^{s}$ is related to the projection onto the model space $\mathcal{U}_{\varepsilon \#}^{s}$, denoted by

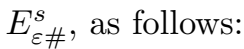

$$
E_{\varepsilon \#}^{s}=\left(\Phi^{T}\right)^{*} E_{\varepsilon}^{s} \Phi^{T}
$$

(the operator $\Phi^{T}$ is defined by (3.1)). This identity can easily be derived from (3.3). Now, it follows that

$$
v^{s}=\left|W^{T}\right|\left(E_{\varepsilon \#}^{s}\right)^{\perp}\left|W^{T}\right| f .
$$

This implies that $v^{s}$ can be obtained within the model (3.2). After that, using $v^{s}$ for different $s$, we may construct $I_{\varepsilon}^{T} W^{T} f$.

For this, we need to find the field

$$
u(\gamma, s):=\lim _{\tau \rightarrow T-s-0} v^{s}(\gamma, \tau)
$$

for every $s \in(0, T)$ and $\gamma \in \Gamma$. Lemma 3 guarantees that this limit exists for all $\gamma \in \sigma_{-}^{s} \cup \sigma_{+}^{s}$ and a.e. $s$. Recall that, for a.e. $s$, the union $\sigma_{-}^{s} \cup \sigma_{+}^{s}$ coincides with $\Gamma$ up to a set of measure zero (this was noted in Subsection 1.2); therefore, the values of $u(\gamma, s)$ are well defined a.e. on the cylinder $\Gamma \times(0, T)$. By formula (5.1) and the definitions of $M_{\eta}^{T}$ and $I_{\eta}^{T}$, the field $u$ equals $\varkappa_{\varepsilon}(\gamma, 0) I_{\varepsilon}^{T} W^{T} f$ a.e. on the cylinder $\Gamma \times(0, T)$, so that we can obtain the image $I_{\varepsilon}^{T} W^{T} f$ if we know the function $\varkappa_{\varepsilon}(\gamma, 0)$. But in Subsection 2.2 we observed that the data of the inverse problem uniquely determine $\varepsilon, \mu$ (and, hence, $\left.\varkappa_{\varepsilon}(\gamma, 0)\right)$ on $\Gamma$.

Thus we obtain a map $f \mapsto I_{\varepsilon}^{T} W^{T} f$, which allows us to construct the model image operator

$$
I_{\varepsilon \#}^{T}:=I_{\varepsilon}^{T} \Phi^{T}
$$

acting from $\mathcal{U}_{\varepsilon \#}^{T}$ to $\mathcal{F}^{T}$. The operator $I_{\varepsilon \#}^{T}$ takes the elements $\left|W^{T}\right| f, f \in \mathcal{F}_{0}^{T}$, to the images $I_{\varepsilon}^{T} W^{T} f$, which can be found by the procedure described above. This gives a bounded operator $I_{\varepsilon \#}^{T}$ defined on a set dense in $\mathcal{U}_{\varepsilon \#}^{T}$ (density follows from (2.7) and (3.1)); thus, it is defined on the entire space.

The model image operator for magnetic fields can be constructed in the same way:

$$
I_{\mu \#}^{T}:=I_{\mu}^{T} \Phi_{m}^{T}
$$

Formula (4.9) is also true for $I_{\varepsilon \#}^{T}, I_{\mu \#}^{T}$. It is easily seen that the identity

$$
\overrightarrow{\mathcal{L}}_{2}\left(\Theta^{T}\right)=\operatorname{Ran} I_{\varepsilon \#}^{T}
$$

determines the pattern $\Theta^{T}$.

\section{§6. RECOVERING THE VELOCITY}

In this section we describe the procedure of recovering the velocity in $\Omega^{T}$ by the model objects (3.2) and $\mathcal{R}_{e \#}^{T}$. Since these are determined by the data of the inverse problem, this procedure will prove that the same data determines the velocity. 
6.1. The Maxwell operator on the pattern. Consider the following operator acting in $\overrightarrow{\mathcal{L}}_{2}\left(\Theta^{T}\right)$ :

$$
\widetilde{\mathcal{R}}_{e}^{T}:=I_{\mu}^{T} \mathcal{R}_{e}^{T}\left(I_{\varepsilon}^{T}\right)^{*}
$$

(recall that $\mathcal{R}_{e}^{T}$ acts as $\mu^{-1}$ curl; see Subsection 3.2). As its domain, we take the set of smooth compactly supported fields in $\overrightarrow{\mathcal{L}}_{2}\left(\Theta^{T}\right)$. We need to show that this definition is consistent. If $v \in \operatorname{Dom} \widetilde{\mathcal{R}}_{e}^{T}$, then $\varkappa_{\varepsilon}^{-1} \pi^{-1} v$ is a smooth compactly supported transversal field in $\Omega^{T} \backslash \omega$; therefore, by formula (4.10) and item $(c)$ of Theorem 3 , we have

$$
\left(I_{\varepsilon}^{T}\right)^{*} v=\left(M_{\varepsilon}^{T}\right)^{*} \varkappa_{\varepsilon}^{-1} \pi^{-1} v \in \mathcal{W}_{\varepsilon}^{T} .
$$

Hence, by Lemma 2 $\left(I_{\varepsilon}^{T}\right)^{*} v \in \operatorname{Dom} \mathcal{R}_{e}^{T}$. Now it remains to recall that the operator $I_{\mu}^{T}$ is bounded.

Using (3.6) and the definitions of $I_{\varepsilon \#}^{T}$ and $I_{\mu \#}^{T}$, one can easily deduce that

$$
\widetilde{\mathcal{R}}_{e}^{T}=I_{\mu \#}^{T} \mathcal{R}_{e \#}^{T}\left(I_{\varepsilon \#}^{T}\right)^{*}
$$

for fields in Dom $\tilde{\mathcal{R}}_{e}^{T}$. This means that the operator $\widetilde{\mathcal{R}}_{e}^{T}$ is determined by the data of the inverse problem.

In [8], an operator similar to $\widetilde{\mathcal{R}}_{e}^{T}$ was considered. More precisely, this was the composition

$$
M_{\mu}^{T} \mu^{-1} \operatorname{curl}\left(M_{\varepsilon}^{T}\right)^{*}: \overrightarrow{\mathcal{L}}_{2, \varepsilon}\left(\Omega^{T}\right) \rightarrow \overrightarrow{\mathcal{L}}_{2, \mu}\left(\Omega^{T}\right) .
$$

One of the results was a formula that described the action of this operator on fields in the set $\vec{C}_{0}^{\infty}\left(\Omega^{T} \backslash \omega\right) \cap \overrightarrow{\mathcal{L}}_{2, \varepsilon}\left(\Omega^{T}\right)$ (see [8, formula (40)]). We shall use this result together with the relation

$$
\widetilde{\mathcal{R}}_{e}^{T}=\pi \varkappa_{\mu}\left(M_{\mu}^{T} \mu^{-1} \operatorname{curl}\left(M_{\varepsilon}^{T}\right)^{*}\right) \varkappa_{\varepsilon}^{-1} \pi^{-1},
$$

which is a consequence of (4.10), to describe the action of $\widetilde{\mathcal{R}}_{e}^{T}$ on $v \in \operatorname{Dom} \widetilde{\mathcal{R}}_{e}^{T}$. It is convenient to introduce the family of operators $\left\{\Lambda_{\eta}^{s}\right\}$ that act on functions on $\Gamma^{s}$ by the rule

$$
\Lambda_{\eta}^{s} \psi:=\left.\left(\widetilde{Q}_{\eta}^{s} \psi\right)\right|_{\Gamma^{s-0}}
$$

(the images are also functions on $\Gamma^{s}$ ). This $\Lambda_{\eta}^{s}$ is known as the Neumann-to-Dirichlet map. We also need a simple identity, which is true outside of $\omega$ for transversal fields:

$$
\operatorname{div} v=c^{-1} \operatorname{div}_{\theta}(c v) .
$$

Now we are ready to write a representation for $\widetilde{\mathcal{R}}_{e}^{T}$ :

$$
\begin{aligned}
& \left(\widetilde{\mathcal{R}}_{e}^{T} v\right)(\cdot, s)=\left(\pi \varkappa_{\mu} \mu^{-1} \Pi \operatorname{curl}\left(\varkappa_{\varepsilon}^{-1} \pi^{-1} v\right)\right)(\cdot, s) \\
& \quad-\pi^{s} \varkappa_{\mu} c^{-1} \mu^{-1} N \nabla_{\theta} \Lambda_{\varepsilon}^{s}\left(\varepsilon^{-1} \operatorname{div}_{\theta}\left(c \varepsilon \varkappa_{\varepsilon}^{-1}\left(\pi^{s}\right)^{-1} v(\cdot, s)\right)\right) \\
& \quad+\pi^{s} \varkappa_{\mu} \nabla_{\theta} \Lambda_{\mu}^{s}\left(\mu^{-1} \operatorname{div}_{\theta}\left(N \varkappa_{\varepsilon}^{-1}\left(\pi^{s}\right)^{-1} v(\cdot, s)\right)\right) \\
& \quad-\pi^{s}\left[\left.\left(\varkappa_{\mu} c^{-1} \mu^{-1} N B_{\varepsilon}^{s} \varepsilon^{-1} \operatorname{curl}\left(\varepsilon c N \varkappa_{\varepsilon}^{-1} \pi^{-1} v\right)+\varkappa_{\mu} B_{\mu}^{s} \mu^{-1} \operatorname{curl}\left(\varkappa_{\varepsilon}^{-1} \pi^{-1} v\right)\right)\right|_{\Gamma^{s-0}}\right]
\end{aligned}
$$

( $\Pi$ is defined in Subsection 1.3), where the argument $(\cdot, s)$ ranges over the pattern $\Theta^{T}$. This representation is valid a.e. on $\sigma_{+}^{s}$ for a.e $s \in(0, T)$. Note that a different formula for $M_{\eta}^{T}$ was used in [8] instead of (4.7) (we have already mentioned this in \$4). This difference is in some additional terms involving projections $B^{s}$ in (6.3), but this does not change the derivation of this formula considerably, so that we do not enter in the details here. Formula (6.3) generalizes the result obtained in [1] to the case where $T \in\left(0, T_{*}\right)$ and $\varepsilon, \mu$ are arbitrary. Note the following important property of the operator $\widetilde{\mathcal{R}}_{e}^{T}$ : unlike $\mu^{-1}$ curl, it is not local because the representation (6.3) contains the nonlocal operators $\Lambda^{s}$ and $B^{s}$. 
6.2. Fiberwise part of the operator $\widetilde{\mathcal{R}}_{e}^{T}$. We say that an operator $\mathcal{A}$ acting on fields on the pattern is fiberwise if

$$
(\mathcal{A} v)(\cdot, s)=\mathcal{A}(s) v(\cdot, s)
$$

where the operators $A(s)$ act in the space of fields on $\sigma_{+}^{s}$. Similarly, the operator $\mathcal{A}$ acting on fields in $\Omega^{T}$ is said to be fiberwise if

$$
\left.(\mathcal{A} v)\right|_{\Gamma^{s}}=\mathcal{A}(s)\left(\left.v\right|_{\Gamma^{s}}\right),
$$

where $A(s)$ act on fields on $\Gamma^{s}$. In the sequel we shall deal with pseudodifferential operators ( $\psi$.d.o.), which are usually defined on complex-valued functions (fields), while we consider real spaces. We say that an operator $\mathcal{A}$ acting in a real space is a $\psi$.d.o. if its extension to the complex space

$$
\widetilde{\mathcal{A}}(\varphi+i \psi):=\mathcal{A} \varphi+i \mathcal{A} \psi
$$

is a $\psi$.d.o.

Following the lines of [1], we consider the fiberwise part of the operator $\widetilde{\mathcal{R}}_{e}^{T}$ to find the optical metric tensor on $\Theta^{T}$.

Lemma 4. Suppose that $v \in \overrightarrow{\mathcal{L}}_{2}\left(\Theta^{T}\right)$ is a smooth compactly supported field and that $s$ satisfies (1.4). Then

$$
\left(\widetilde{\mathcal{R}}_{e}^{T} v\right)(\cdot, s)=N \frac{\partial v}{\partial s}(\cdot, s)+A(s) v(\cdot, s)
$$

where $A(s)$ is the family of linear operators on tangential fields on $\sigma_{+}^{s}$ defined by the formula

$$
\begin{aligned}
A(s) u= & -\pi^{s} \varkappa_{\mu} c^{-1} \mu^{-1} N \nabla_{\theta} \Lambda_{\varepsilon}^{s}\left(\varepsilon^{-1} \operatorname{div}_{\theta}\left(c \varepsilon \varkappa_{\varepsilon}^{-1}\left(\pi^{s}\right)^{-1} u\right)\right) \\
& +\pi^{s} \varkappa_{\mu} \nabla_{\theta} \Lambda_{\mu}^{s}\left(\mu^{-1} \operatorname{div}_{\theta}\left(N \varkappa_{\varepsilon}^{-1}\left(\pi^{s}\right)^{-1} u\right)\right)+A_{0}(s) u,
\end{aligned}
$$

where $A_{0}(s)$ is a $\psi$.d.o. of order 0 .

Proof. To prove this, we analyze the terms in (6.3). The second and third terms are obviously fiberwise and we can include them in $A(s)$. Now we check that the last two terms with the projections $B^{s}$ are also fiberwise. For example, consider the last term. Choose an orthonormal basis $\left\{\alpha_{n}^{s}\right\}$ in the space $J_{\mu}^{s} \ominus \mathcal{U}_{\mu}^{s}$. For $\alpha^{s}:=B_{\mu}^{s} \mu^{-1} \operatorname{curl}\left(\varkappa_{\varepsilon}^{-1} \pi^{-1} v\right)$, we have

$$
\alpha^{s}=\sum_{n}\left(\operatorname{curl}\left(\varkappa_{\varepsilon}^{-1} \pi^{-1} v\right), \alpha_{n}^{s}\right)_{\vec{L}_{2}\left(\Omega^{s}\right)} \alpha_{n}^{s} .
$$

But the terms of this sum depend only on $v(\cdot, s)$, which follows from the formula

$$
\left(\operatorname{curl}\left(\varkappa_{\varepsilon}^{-1} \pi^{-1} v\right), \alpha_{n}^{s}\right)_{\vec{L}_{2}\left(\Omega^{s}\right)}=\int_{\Gamma^{s}}\left\langle N \varkappa_{\varepsilon}^{-1} \pi^{-1} v, \alpha_{n}^{s}\right\rangle d \Gamma^{s}
$$

(we have integrated by parts and employed the fact that $\operatorname{curl} \alpha_{n}^{s}=0$ and $\left.\left(\alpha_{n}^{s}\right)_{\theta}\right|_{\Gamma}=0$; see (2.2) $)$. Hence, $\left.\alpha^{s}\right|_{\Gamma^{s-0}}$ is also determined by the restriction $v(\cdot, s)$. Moreover, the resulting formula shows that the map $\left.v(\cdot, s) \mapsto \alpha^{s}\right|_{\Gamma^{s-0}}$ is an integral operator with smooth kernel, i.e., a $\psi$.d.o. of order $-\infty$.

Now we turn to the first term in (6.3). We use the representation of curl in local coordinates:

$$
(\operatorname{curl} v)^{j}=\rho^{-1 / 2} \sum_{m, n, k} \epsilon^{j m n} \frac{\partial}{\partial x^{m}}\left(g_{n k} v^{k}\right), \quad \rho:=\operatorname{det}\left\{g_{m n}\right\}
$$

$\left(\epsilon^{j m n}\right.$ is the parity of the permutation $\left.(1,2,3) \mapsto(j, m, n)\right)$. In the semigeodesic coordinates $\left(\gamma^{1}, \gamma^{2}, \tau\right)$, where $\gamma^{1}, \gamma^{2}$ are the coordinates of a point $\gamma(x) \in \Gamma$, the metric tensor 
has the form (1.3). Note that the action of the operator $\Pi$ in such coordinates reduces to nullifying the third component of the vector. Then for a transversal field $v$ we have

$$
\begin{aligned}
& (\Pi \operatorname{curl} v)^{1}=-\rho^{-1 / 2} \sum_{\alpha}\left(g_{2 \alpha} \frac{\partial v^{\alpha}}{\partial \tau}+\frac{\partial g_{2 \alpha}}{\partial \tau} v^{\alpha}\right), \\
& (\Pi \operatorname{curl} v)^{2}=\rho^{-1 / 2} \sum_{\alpha}\left(g_{1 \alpha} \frac{\partial v^{\alpha}}{\partial \tau}+\frac{\partial g_{1 \alpha}}{\partial \tau} v^{\alpha}\right), \quad(\Pi \operatorname{curl} v)^{3}=0 .
\end{aligned}
$$

In semigeodesic coordinates, we have $\left\{\nu^{j}\right\}=\left(0,0, c^{-1}\right)$. Using the formula

$$
(\nu \times v)^{j}=\rho^{-1 / 2} \sum_{k, l, m, n} \epsilon^{j m n} g_{m k} \nu^{k} g_{n l} v^{l}
$$

for the vector product in local coordinates, we obtain

$$
(N v)^{1}=-\rho^{-1 / 2} c \sum_{\alpha} g_{2 \alpha} v^{\alpha}, \quad(N v)^{2}=\rho^{-1 / 2} c \sum_{\alpha} g_{1 \alpha} v^{\alpha}, \quad(N v)^{3}=0 .
$$

We introduce yet another operation $D_{\tau}$ on smooth vector fields in $\Omega^{T}: D_{\tau}$ acts as the covariant derivative in the optical metric along the vector field $c \nu$. The action of $D_{\tau}$ on a transversal field $v$ in semigeodesic coordinates looks like this:

$$
\left(D_{\tau} v\right)^{\alpha}=\frac{\partial v^{\alpha}}{\partial \tau}+\sum_{m} \Gamma_{3 m}^{\alpha} v^{m}, \quad\left(D_{\tau} v\right)^{3}=0
$$

where $\Gamma_{k m}^{j}$ are the Christoffel symbols in optical metric. The second relation follows from the second formula in (1.3) and expressions for Christoffel symbols.

Combining (6.7), (6.8), and (6.9) we obtain an identity valid for transversal fields:

$$
\Pi \operatorname{curl}=c^{-1} N D_{\tau}+A,
$$

where $A$ is a pointwise linear transformation with smooth coefficients. In what follows, we shall use two relations, which can be derived from properties of the covariant derivative and parallel transport:

$$
\pi N=N \pi, \quad \pi D_{\tau}=\frac{\partial}{\partial \tau} \pi
$$

Using the formula for $\Pi$ curl, we obtain

$$
\pi \Pi \operatorname{curl} \pi^{-1}=c^{-1} N \frac{\partial}{\partial \tau}+\pi A \pi^{-1} .
$$

Clearly, the first term in (6.3) differs from

$$
\pi \varkappa_{\mu} \mu^{-1} \varkappa_{\varepsilon}^{-1} \Pi \operatorname{curl}\left(\pi^{-1} v\right)=\varkappa_{\mu} \mu^{-1} \varkappa_{\varepsilon}^{-1}\left(c^{-1} N \frac{\partial}{\partial \tau}+\pi A \pi^{-1}\right) v
$$

by the action of some pointwise linear transformation on $v$. But the scalar factor in front of the bracket equals $c$, which follows from the definition of $\varkappa_{\varepsilon}, \varkappa_{\mu}$ (see (4.8)) and relation (0.2). Therefore, we have

$$
\pi \varkappa_{\mu} \mu^{-1} \Pi \operatorname{curl}\left(\varkappa_{\varepsilon}^{-1} \pi^{-1} v\right)=N \frac{\partial v}{\partial \tau}+\widetilde{A} v
$$

where $\widetilde{A}$ is also a pointwise operator. Together with the analysis of the other terms in (6.3), this proves (6.4). 
6.3. Principal symbol of the operator $\widetilde{\mathcal{R}}_{e}^{T}$. Note that. for a.e. $s$, the first two terms in the formula for $A(s)$ in Lemma 4 are $\psi$.d.o. of the first order acting on tangential fields in $\sigma_{+}^{s}$. This follows from the fact that the operators $\Lambda_{\varepsilon}^{s}$ and $\Lambda_{\mu}^{s}$ associated with the boundary-value problem of the form (4.1) in $\Omega^{s}$ are scalar $\psi$.d.o. of order -1 on $\Gamma^{s} \backslash \omega$. This is well known for smooth $\Gamma^{s}$ and is also true if we restrict the operator to functions in $\Gamma^{s} \backslash \omega$ provided that (1.4) is satisfied.

Fix local coordinates $\left(\gamma^{1}, \gamma^{2}\right) \in D \subset \mathbb{R}^{2}$ on the surface $\sigma_{+}^{s}$. Let $\left\{A_{\beta}^{\alpha}(s)\right\}$ be the matrix representing the operator $A(s)$ in these coordinates:

$$
(A(s) v)^{\alpha}=\sum_{\beta} A_{\beta}^{\alpha}(s) v^{\beta} .
$$

The $A_{\beta}^{\alpha}(s)$ are scalar $\psi$.d.o. in $D$. By a symbol of a $\psi$.d.o. we mean the symbol of some proper $\psi$.d.o. that approximates the given one up to a smoothing operator. The symbol is defined up to terms of order $-\infty$. We denote the symbol of $A_{\beta}^{\alpha}(s)$ by $a_{\beta}^{\alpha}\left(s ; \gamma^{1}, \gamma^{2}, k\right)\left(k \in \mathbb{R}^{2}\right)$. Let $h^{m n}$ be a representation of the tensor $h$ in the semigeodesic coordinates $\left(\gamma^{1}, \gamma^{2}, \tau\right)$. The next lemma establishes a relationship between the matrix symbol $a(s)=\left\{a_{\beta}^{\alpha}(s)\right\}$ and the values $h^{\alpha \beta}$ for $\tau=s(\alpha, \beta=1,2)$.

Lemma 5. If $s \in(0, T)$ satisfies (1.4), then

$$
\operatorname{det} a\left(s ; \gamma^{1}, \gamma^{2}, k\right)=-\sum_{\alpha, \beta} h^{\alpha \beta}\left(\gamma^{1}, \gamma^{2}, s\right) k_{\alpha} k_{\beta} \cdot(1+o(1)), \quad k \rightarrow \infty
$$

Proof. First, consider the following operator acting on tangential fields on $\Gamma^{s} \backslash \omega$ :

$$
\widehat{A}(s) u:=-\varkappa_{\mu} c^{-1} \mu^{-1} N \nabla_{\theta} \Lambda_{\varepsilon}^{s}\left(\varepsilon^{-1} \operatorname{div}_{\theta}\left(c \varepsilon \varkappa_{\varepsilon}^{-1} u\right)\right)+\varkappa_{\mu} \nabla_{\theta} \Lambda_{\mu}^{s}\left(\mu^{-1} \operatorname{div}_{\theta}\left(N \varkappa_{\varepsilon}^{-1} u\right)\right) .
$$

The mapping

$$
\left(\gamma^{1}, \gamma^{2}\right) \mapsto x\left(\gamma\left(\gamma^{1}, \gamma^{2}\right), s\right) \in \Gamma^{s}
$$

takes $\left(\gamma^{1}, \gamma^{2}\right)$ to coordinates on $\Gamma^{s}$. In these coordinates, the operator $\widehat{A}(s)$ is represented by a matrix of scalar operators, and the corresponding matrix symbol is $\widehat{a}(s)=\left\{\widehat{a}_{\beta}^{\alpha}(s)\right\}$ ( $\alpha$ is a row number, $\beta$ is a column number). Clearly, the coordinate representation $\left\{g_{\alpha \beta}\left(\gamma^{1}, \gamma^{2}\right)\right\}$ of the (2-dimensional) metric tensor on $\Gamma^{s}$ in the coordinates $\left(\gamma^{1}, \gamma^{2}\right)$ coincides with the representation $\left\{g_{\alpha \beta}\left(\gamma^{1}, \gamma^{2}, s\right)\right\}$ in semigeodesic coordinates. The same can be said about the tensor $h_{\alpha \beta}$. Put $G\left(\gamma^{1}, \gamma^{2}\right):=\left\{g_{\alpha \beta}\left(\gamma^{1}, \gamma^{2}\right)\right\}$ (this is a $(2 \times 2)$-matrix). By formulas (6.8), the matrix of the operator $N$ is equal to

$$
\rho^{-1 / 2} c\left(\begin{array}{cc}
0 & -1 \\
1 & 0
\end{array}\right) G=\frac{1}{\sqrt{\operatorname{det} G}}\left(\begin{array}{cc}
0 & -1 \\
1 & 0
\end{array}\right) G
$$

We also need coordinate representations of the operators $\nabla_{\theta}$ and $\operatorname{div}_{\theta}$ :

$$
G^{-1}\left(\begin{array}{l}
\partial / \partial \gamma^{1} \\
\partial / \partial \gamma^{2}
\end{array}\right), \quad \frac{1}{\sqrt{\operatorname{det} G}}\left(\frac{\partial}{\partial \gamma^{1}}(\sqrt{\operatorname{det} G} \cdot), \frac{\partial}{\partial \gamma^{2}}(\sqrt{\operatorname{det} G} \cdot)\right) .
$$

The principal symbols of these operators are of the form

$$
G^{-1}\left(\begin{array}{l}
i k_{1} \\
i k_{2}
\end{array}\right), \quad\left(i k_{1}, i k_{2}\right)
$$

Moreover, the Neumann-to-Dirichlet maps $\Lambda_{\varepsilon}^{s}, \Lambda_{\mu}^{s}$ have principal symbol $|k|_{g}^{-1}$, where

$$
|k|_{g}:=\left(\sum_{\alpha, \beta} g^{\alpha \beta}\left(\gamma^{1}, \gamma^{2}\right) k_{\alpha} k_{\beta}\right)^{1 / 2}
$$


To calculate the principal part of the symbol $\widehat{a}(s)$, we collect the scalar factors in each term of the formula for $\widehat{A}(s)$. For both terms, the resulting factor equals $c$. Now we write the principal part of the symbol $\hat{a}(s)$ :

$$
\begin{aligned}
-\frac{c}{\sqrt{\operatorname{det} G}}\left(\begin{array}{cc}
0 & -1 \\
1 & 0
\end{array}\right) & \left(\begin{array}{l}
i k_{1} \\
i k_{2}
\end{array}\right) \frac{1}{|k|_{g}}\left(i k_{1}, i k_{2}\right)+c G^{-1}\left(\begin{array}{c}
i k_{1} \\
i k_{2}
\end{array}\right) \frac{1}{|k|_{g}}\left(i k_{1}, i k_{2}\right) \frac{1}{\sqrt{\operatorname{det} G}}\left(\begin{array}{cc}
0 & -1 \\
1 & 0
\end{array}\right) G \\
= & \frac{c}{|k|_{g} \sqrt{\operatorname{det} G}}\left[\left(\begin{array}{cc}
-k_{1} k_{2} & -\left(k_{2}\right)^{2} \\
\left(k_{1}\right)^{2} & k_{1} k_{2}
\end{array}\right) G^{-1}-G^{-1}\left(\begin{array}{cc}
k_{1} k_{2} & -\left(k_{1}\right)^{2} \\
\left(k_{2}\right)^{2} & -k_{1} k_{2}
\end{array}\right)\right] G .
\end{aligned}
$$

Direct calculations show that the matrix in square brackets is equal to

$$
\left(\begin{array}{cc}
-2\left(g^{11} k_{1} k_{2}+g^{12}\left(k_{2}\right)^{2}\right) & g^{11}\left(k_{1}\right)^{2}-g^{22}\left(k_{2}\right)^{2} \\
g^{11}\left(k_{1}\right)^{2}-g^{22}\left(k_{2}\right)^{2} & 2\left(g^{12}\left(k_{1}\right)^{2}+g^{22} k_{1} k_{2}\right)
\end{array}\right),
$$

and its determinant is $-|k|_{g}^{4}$. Using the expression obtained for the principal symbol of the operator $\widehat{A}(s)$, we arrive at the relation

$$
\operatorname{det} \widehat{a}(s)=-c^{2}|k|_{g}^{2} \cdot(1+o(1))=-\sum_{\alpha, \beta} h^{\alpha \beta} k_{\alpha} k_{\beta} \cdot(1+o(1)), \quad k \rightarrow \infty
$$

(in the second identity we used the formula $h^{\alpha \beta}=c^{2} g^{\alpha \beta}$, see (1.1) and (1.3)). The above calculations also show that $\widehat{A}(s)$ is a first-order operator.

Now we turn to the operator $A(s)$. Let $p=\left\{p_{\beta}^{\alpha}\right\}$ be the matrix of the operator $\pi^{s}$ :

$$
\left(\pi^{s} u\right)^{\alpha}=\sum_{\beta} p_{\beta}^{\alpha} u^{\beta}
$$

On the left, the coordinate representation of the field $\pi^{s} u$ on $\Gamma$ in the coordinates $\left(\gamma^{1}, \gamma^{2}\right)$ is written, and $u^{\beta}$ on the right is a representation of the field $u$ on $\Gamma^{s}$ in the coordinates (6.11).

Since $A(s)=\pi^{s}\left(\widehat{A}(s)+A_{0}(s)\right)\left(\pi^{s}\right)^{-1}$, modulo terms of lower order the symbol $a(s)$ is equal to $p \widehat{a}(s) p^{-1}$ (we ignore the contribution of $A_{0}(s)$ to the symbol, because it is a 0 -order operator). Together with (6.12), this finishes the proof.

On the pattern, the map $i^{-1}$ induces an optical metric tensor pulled back from $\Omega^{T} \backslash \omega$. This tensor can be recovered a.e. (thus, everywhere, because it is smooth) on $\Theta^{T}$ by using formula (6.10) for different local coordinates on $\Gamma$ and varying $s \in(0, T)$. For this we need the operators $A(s)$ occurring in (6.4), but they can be extracted from $\widetilde{\mathcal{R}}_{e}^{T}$. By Theorem [1] the function $c$ in $\Omega^{T}$ can be recovered by the optical metric tensor on the pattern.

In [2], the second order operator $I_{\varepsilon}^{T} \varepsilon^{-1} \operatorname{curl} \mu^{-1} \operatorname{curl}\left(I_{\varepsilon}^{T}\right)^{*}$ was used (instead of $\left.\widetilde{\mathcal{R}}_{e}^{T}\right)$ to recover the velocity. In the case of arbitrary $T$, the domain of this operator may fail to contain all smooth compactly supported tangential fields on the pattern, because when the equidistant surfaces are nonsmooth, the field $\left(I_{\varepsilon}^{T}\right)^{*} v$ may also be nonsmooth and its double curl may fail to belong to $\vec{L}_{2}\left(\Omega^{T}\right)$. For this reason, we invoked the first order operator $\widetilde{\mathcal{R}}_{e}^{T}$, which will also be used below to recover $\varepsilon, \mu$. Note that, compared to the case of regular semigeodesic coordinates in $\Omega^{T}$ (as considered in [2]), the most significant difficulty of recovering $c$ in the general case was to generalize the definition of the image operator (see Subsection 4.2).

\section{$\S 7$ SPACES $J_{\varepsilon}\left\langle\Omega^{r}[x]\right\rangle$ AND $J_{\varepsilon \#}\left\langle\Omega^{r}[x]\right\rangle$}

To finish the proof of Theorem 2 it remains to show the uniqueness of the coefficients $\varepsilon$ and $\mu$. For this, we use some constructions from [4]. 
7.1. Control on a part of the boundary. Let $\sigma$ be an open subset of $\Gamma$. We introduce a set of smooth delayed controls supported on $\sigma$ :

$$
\mathcal{F}_{0}^{T, s}[\sigma]:=\left\{f \in \mathcal{F}_{0}^{T, s} \mid \operatorname{supp} f \subset \sigma \times(T-s, T]\right\} .
$$

Obviously, $\mathcal{F}_{0}^{T, s}=\mathcal{F}_{0}^{T, s}[\Gamma]$. The metric $r$-neighborhood of a set $K \subset \bar{\Omega}$ will be defined as

$$
\Omega^{r}[K]:=\left\{x \in \Omega \mid \operatorname{dist}_{c}(x, K)<r\right\} .
$$

The neighborhood $\Omega^{r}[\{x\}]$ of a point $x$ is denoted by $\Omega^{r}[x]$. Let $\sigma_{\delta}(\gamma)$ be a $\delta$-neighborhood of a point $\gamma \in \Gamma$ on the surface $\Gamma$ (with respect to the optical metric). For a point $(\gamma, s) \in \Theta^{T}$ of the pattern, define a family of open subsets of $\Omega$ :

$$
a_{\gamma}^{s, \delta}:=\Omega^{s}\left[\sigma_{\delta}(\gamma)\right] \backslash \overline{\Omega^{s-\delta}}, \quad \delta>0 .
$$

This family is monotone increasing with $\delta$; the sets $a_{\gamma}^{s, \delta}$ tend to the point $x(\gamma, s)$ as $\delta \rightarrow 0$, more precisely,

$$
\bigcap_{\delta>0} \overline{a_{\gamma}^{s, \delta}}=\{x(\gamma, s)\} .
$$

Next, we introduce the space of solenoidal fields vanishing outside an open set $U \subset \Omega$ :

$$
J_{\varepsilon}\langle U\rangle:=\operatorname{clos}\left\{y \in \vec{C}_{0}^{\infty}(U) \mid \operatorname{div}(\varepsilon y)=0\right\} .
$$

Lemma 6. Suppose $U$ is a domain with Lipschitz boundary, $\bar{U} \subset \Omega$. Then any field $y \in J_{\varepsilon}\langle\Omega\rangle$ such that $\operatorname{supp} y \subset \bar{U}$ belongs to $J_{\varepsilon}\langle U\rangle$.

Proof. Suppose the domain $\tilde{U} \subset \Omega$ contains $\bar{U}$. We may assume that $\tilde{U}$ is a domain with Lipschitz boundary; hence, the Helmholtz decomposition is applicable:

$$
\vec{L}_{2, \varepsilon}(\tilde{U})=\left\{\nabla \widetilde{\varphi} \mid \tilde{\varphi} \in H^{1}(\tilde{U})\right\} \oplus J_{\varepsilon}\langle\tilde{U}\rangle
$$

(see [12]). The function $\varphi \in H^{1}(U)$ has some extension $\tilde{\varphi}$ to $\widetilde{U}$. Since the field $y$ admits approximation in $\vec{L}_{2, \varepsilon}(\tilde{U})$ by fields $y_{(n)} \in \vec{C}_{0}^{\infty}(\tilde{U})$ with $\operatorname{div}\left(\varepsilon y_{(n)}\right)=0$, we have

$$
(y, \nabla \varphi)_{\vec{L}_{2, \varepsilon}(U)}=(y, \nabla \widetilde{\varphi})_{\vec{L}_{2, \varepsilon}(\widetilde{U})}=\lim _{n \rightarrow \infty}\left(y_{(n)}, \nabla \widetilde{\varphi}\right)_{\vec{L}_{2, \varepsilon}(\widetilde{U})}=0
$$

(the last identity follows from the orthogonal decomposition (7.2)). Now it follows that $y \in J_{\varepsilon}\langle U\rangle$, because (7.2) holds true for the domain $U$ as well.

Next we prove that if $U$ is homeomorphic to a ball, then

$$
J_{\varepsilon}\langle U\rangle \subset \mathcal{U}_{\varepsilon}^{T} .
$$

We shall establish this inclusion for elements $y \in \vec{C}_{0}^{\infty}(U) \cap J_{\varepsilon}\langle U\rangle$. Clearly, we have $y \in J_{\varepsilon}^{T}$, so that it suffices to prove that $(y, \alpha)_{\vec{L}_{2, \varepsilon}(U)}=0$ for any $\alpha \in J_{\varepsilon}^{T} \ominus \mathcal{U}_{\varepsilon}^{T}$. Since $\operatorname{curl} \alpha=0$ by (2.2), the restriction of $\alpha$ to the set $U$ is equal to the gradient $\nabla \varphi$ of a smooth function. Therefore,

$$
(y, \alpha)_{\vec{L}_{2, \varepsilon}(U)}=(y, \nabla \varphi)_{\vec{L}_{2, \varepsilon}(U)}=0 .
$$

The solution $\{e, h\}$ corresponding to a control $f \in \mathcal{F}_{0}^{T, s}[\sigma]$ satisfies

$$
\operatorname{supp}\{e(\cdot, T), h(\cdot, T)\} \subset \overline{\Omega^{s}[\sigma]},
$$

which follows from the finiteness of the velocity of wave propagation. A nontrivial fact, which was proved in [4], is that, for any open set homeomorphic to a ball, the inclusion

$$
J_{\varepsilon}\langle U\rangle \subset \operatorname{clos}_{J_{\varepsilon}^{T}} W^{T} \mathcal{F}_{0}^{T, s}[\sigma]
$$


holds true. This is a generalization of the approximate controllability property (2.8). In [4. Lemma 4], an important consequence of this inclusion was obtained:

$$
J_{\varepsilon}\left\langle a_{\gamma}^{s, \delta}\right\rangle=\left(\mathcal{U}_{\varepsilon}^{s} \ominus \mathcal{U}_{\varepsilon}^{s-\delta}\right) \cap \operatorname{clos}_{J_{\varepsilon}^{T}} W^{T} \mathcal{F}_{0}^{T, s}\left[\sigma_{\delta}(\gamma)\right]
$$

for a fixed point $(\gamma, s) \in \Theta^{T}$ and sufficiently small $\delta>0$.

7.2. The problem with volume sources. We introduce an antiselfadjoint operator in the space $\mathcal{U}_{\varepsilon}^{T} \oplus \mathcal{U}_{\mu}^{T}$ :

$$
\mathcal{M}^{T}:=\left(\begin{array}{cc}
0 & \left(\mathcal{R}_{e}^{T}\right)^{*} \\
-\mathcal{R}_{e}^{T} & 0
\end{array}\right)
$$

$\left(\mathcal{R}_{e}^{T}\right.$ is defined in 3.2). Unlike $\mathcal{R}_{e}^{T}$, the operator $\left(\mathcal{R}_{e}^{T}\right)^{*}$ is not local. The domain of $\left(\mathcal{R}_{e}^{T}\right)^{*}$ contains the fields $z \in \mathcal{U}_{\mu}^{T}$ such that $\operatorname{curl} z \in \vec{L}_{2}\left(\Omega^{T}\right)$ and $\left(\mathcal{R}_{e}^{T}\right)^{*} z=E_{\varepsilon}^{T} \varepsilon^{-1} \operatorname{curl} z$. It is the projection $E_{\varepsilon}^{T}$ onto $\mathcal{U}_{\varepsilon}^{T}$ that makes the operator nonlocal. On the other hand, the definition of the space $\mathcal{U}_{\varepsilon}^{T}$ shows that

$$
E_{\varepsilon}^{T} \varepsilon^{-1} \operatorname{curl} z=\varepsilon^{-1} \operatorname{curl} z, \quad z \in \vec{C}_{0}^{\infty}\left(\Omega^{T} \cup \Gamma\right),
$$

implying that $\left(\mathcal{R}_{e}^{T}\right)^{*}$ acts as a local operator on fields that are compactly supported in $\Omega^{T} \cup \Gamma$. Next, we consider the following problem for functions $E(t), H(t)$ on the interval $[0, T]$ with values in $\mathcal{U}_{\varepsilon}^{T}, \mathcal{U}_{\mu}^{T}$, respectively:

$$
\begin{gathered}
\left(\begin{array}{c}
E_{t} \\
H_{t}
\end{array}\right)-\mathcal{M}^{T}\left(\begin{array}{c}
E \\
H
\end{array}\right)=\left(\begin{array}{c}
K \\
0
\end{array}\right), \\
\left.E\right|_{t=0}=\left.H\right|_{t=0}=0,
\end{gathered}
$$

where $K \in L_{2}\left([0, T] ; \mathcal{U}_{\varepsilon}^{T}\right)$. The control operator

$$
W_{\mathrm{vol}}^{T}: K \mapsto E(\cdot, T)
$$

for (7.6) is continuous from $L_{2}\left([0, T] ; \mathcal{U}_{\varepsilon}^{T}\right)$ to $\mathcal{U}_{\varepsilon}^{T}$. By analogy with the delayed controls $\mathcal{F}_{0}^{T, s}[\sigma]$ acting on the part of the boundary, we consider system (7.6) with a delayed $K$ acting in $\bar{U}\left(U\right.$ is an open subset of $\left.\Omega^{T}\right)$ : $\operatorname{supp} K \subset \bar{U} \times[T-r, T], r \in[0, T]$. It can be shown that if $\bar{U}, \overline{\Omega^{r}[U]} \subset \Omega^{T}$, then the solution vanishes outside of $\Omega^{r}[U]$ at $t=T$.

Introduce the subspace of $\mathcal{U}_{\varepsilon}^{T}$ spanned by all possible values of $E$ at $t=T$ corresponding to delayed sources supported on $\bar{U}$ :

$$
\mathcal{U}_{\varepsilon}^{r}[U]:=\cos _{J_{\varepsilon}^{T}}\left\{W_{\mathrm{vol}}^{T} K \mid K \in L_{2}\left([0, T] ; \mathcal{U}_{\varepsilon}^{T}\right), \operatorname{supp} K \subset \bar{U} \times[T-r, T]\right\} .
$$

The following proposition easily follows from Theorem 3 in [4].

Proposition 2. Let $U$ be an open subset of $\Omega^{T}$, and let a number $r>0$ be such that

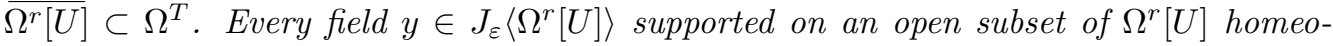
morphic to a ball belongs to $\mathcal{U}_{\varepsilon}^{r}[U]$.

We can use this proposition with $U=a_{\gamma}^{s, \delta}$ if $\delta$ is sufficiently small. If for some $r>0$ the set $\Omega^{r}[x(\gamma, s)]$ is homeomorphic to a ball and its closure is contained in $\Omega^{T}$, then

$$
\bigcap_{\delta>0} \mathcal{U}_{\varepsilon}^{r}\left[a_{\gamma}^{s, \delta}\right]=J_{\varepsilon}\left\langle\Omega^{r}[x]\right\rangle
$$

where $x=x(\gamma, s)$. Indeed, Proposition 2 can be applied to any field $y \in \vec{C}_{0}^{\infty}\left(\Omega^{r}[x]\right)$ with $\operatorname{div}(\varepsilon y)=0$ if we put $U=a_{\gamma}^{s, \delta}$. Therefore, the right-hand side of (17.8) is contained in the left-hand side. To prove the reverse inclusion, note that (7.1) and the monotonicity of the sets $a_{\gamma}^{s, \delta}$ with respect to $\delta$ imply that the diameters of these sets tend to zero as $\delta \rightarrow 0$. Therefore,

$$
\bigcap_{\delta>0} \overline{\Omega^{r}\left[a_{\gamma}^{s, \delta}\right]}=\overline{\Omega^{r}[x]}
$$


Then supp $y \subset \overline{\Omega^{r}[x]}$ for any element $y$ belonging to the left-hand side of (7.8). Combined with the condition $\operatorname{div}(\varepsilon y)=0$ and Lemma 6 , this implies that $y \in J_{\varepsilon}\left\langle\Omega^{r}[x]\right\rangle$.

Since the space $J_{\varepsilon}\left\langle\Omega^{r}[x]\right\rangle$ on the right-hand side of (7.8) is contained in $\mathcal{U}_{\varepsilon}^{T}$, it has a corresponding model space $\left(\Phi^{T}\right)^{*} J_{\varepsilon}\left\langle\Omega^{r}[x]\right\rangle \subset \mathcal{U}_{\varepsilon \#}^{T}$. In the remaining part of the section we show how to construct this space for sufficiently small $r$ by using the model (3.2) and the operator $\mathcal{R}_{e \#}^{T}$. For this, we employ the relations obtained above. We introduce a model counterpart of the control operator

$$
W_{\mathrm{vol} \#}^{T} K_{\#}:=\left(\Phi^{T}\right)^{*} W_{\mathrm{vol}}^{T} K
$$

where $K_{\#}$ and $K$ are related by $K(\cdot, t)=\Phi^{T} K_{\#}(\cdot, t)$, and the operator

$$
\mathcal{M}_{\#}^{T}:=\left(\Phi^{T} \oplus \Phi_{m}^{T}\right)^{*} \mathcal{M}^{T}\left(\Phi^{T} \oplus \Phi_{m}^{T}\right) .
$$

The following problem for functions $E_{\#}(t), H_{\#}(t)$ on the interval $[0, T]$ with values (respectively) in $\mathcal{U}_{\varepsilon \#}^{T}, \mathcal{U}_{\mu \#}^{T}$ is similar to (7.6):

$$
\begin{gathered}
\left(\begin{array}{c}
\left(E_{\#}\right)_{t} \\
\left(H_{\#}\right)_{t}
\end{array}\right)-\mathcal{M}_{\#}^{T}\left(\begin{array}{c}
E_{\#} \\
H_{\#}
\end{array}\right)=\left(\begin{array}{c}
K_{\#} \\
0
\end{array}\right), \\
\left.E_{\#}\right|_{t=0}=\left.H_{\#}\right|_{t=0}=0
\end{gathered}
$$

where $K_{\#} \in L_{2}\left([0, T] ; \mathcal{U}_{\varepsilon \#}^{T}\right)$. The action of the operator $W_{\mathrm{vol} \#}^{T}$ is described as follows:

$$
W_{\mathrm{vol} \#}^{T}: K_{\#} \mapsto E_{\#}(\cdot, T) .
$$

Since $\mathcal{M}_{\#}^{T}$ can easily be expressed via $\mathcal{R}_{e \#}^{T}$, the operator $W_{\text {vol\# }}^{T}$ also can be obtained from $\mathcal{R}_{e \#}^{T}$.

For an open set $U$ satisfying $\bar{U} \subset \Omega^{T}$ and homeomorphic to a ball, let $J_{\varepsilon \#}\langle U\rangle:=$ $\left(\Phi^{T}\right)^{*} J_{\varepsilon}\langle U\rangle$. Let $(\gamma, s)$ be the semigeodesic coordinates of a point $x$. For small $\delta$, as in (17.4), we have

$$
J_{\varepsilon \#}\left\langle a_{\gamma}^{s, \delta}\right\rangle=\left(\mathcal{U}_{\varepsilon \#}^{s} \ominus \mathcal{U}_{\varepsilon \#}^{s-\delta}\right) \cap \operatorname{clos}_{\mathcal{U}_{\varepsilon \#}^{T}}\left|W^{T}\right| \mathcal{F}_{0}^{T, s}\left[\sigma_{\delta}(\gamma)\right] .
$$

This allows us to obtain the spaces on the left-hand side by the model (3.2). After that, we can pass to the spaces $\mathcal{U}_{\varepsilon \#}^{r}\left[a_{\gamma}^{s, \delta}\right]:=\left(\Phi^{T}\right)^{*} \mathcal{U}_{\varepsilon}^{r}\left[a_{\gamma}^{s, \delta}\right]$. To do this, we note that in the definition (7.7) for $U=a_{\gamma}^{s, \delta}$, the sources $K$ belong to $L_{2}\left([0, T] ; J_{\varepsilon}\left\langle a_{\gamma}^{s, \delta}\right\rangle\right)$ (this follows from Lemma (6) and vanish on the interval $[0, T-r)$; hence, we can construct $\mathcal{U}_{\varepsilon \#}^{r}\left[a_{\gamma}^{s, \delta}\right]$ with the help of the formula

$$
\mathcal{U}_{\varepsilon \#}^{r}\left[a_{\gamma}^{s, \delta}\right]=\operatorname{clos}_{\mathcal{U}_{\varepsilon \#}^{T}}\left\{W_{\text {vol\# }}^{T} K_{\#} \mid K_{\#} \in L_{2}\left([0, T] ; J_{\varepsilon \#}\left\langle a_{\gamma}^{s, \delta}\right\rangle\right), \operatorname{supp} K_{\#} \subset[T-r, T]\right\} .
$$

Now it remains to obtain the space $J_{\varepsilon \#}\left\langle\Omega^{r}[x]\right\rangle$ by using the model analog of (7.8).

\section{§8. Projections onto $J_{\varepsilon}\left\langle\Omega^{r}[x]\right\rangle$ For small $r$}

For an open set $U \subset \Omega$, we denote by $P_{\varepsilon}\langle U\rangle$ the projection onto $J_{\varepsilon}\langle U\rangle$ acting in $J_{\varepsilon}\langle\Omega\rangle$. Suppose $\partial U \in C^{\infty}$ and denote by $n$ the outward unit normal to $\partial U$. In Subsection 4.1 we described the projection $P_{\varepsilon}^{s}$ in terms of the boundary-value problem (4.1). Similar arguments lead to the formula

$$
\left.P_{\varepsilon}\langle U\rangle y\right|_{U}=\left.(y-\nabla p)\right|_{U},
$$

where $y \in \vec{C}^{\infty}(\bar{\Omega}) \cap J_{\varepsilon}\langle\Omega\rangle$, and $p \in H^{1}(U)$ is the solution of the following boundary-value problem:

$$
\operatorname{div}(\varepsilon \nabla p)=0,\left.\quad \frac{\partial p}{\partial n}\right|_{\partial U}=\left.\langle y, n\rangle\right|_{\partial U} .
$$

The following lemma describes the behavior of the projections $P_{\varepsilon}\left\langle\Omega^{r}[x]\right\rangle$ as $r \rightarrow 0$. 
Lemma 7. Let $x_{0} \in \Omega$. For $y \in \vec{C}^{\infty}(\Omega) \cap J_{\varepsilon}\langle\Omega\rangle$, we have

$$
\left(P_{\varepsilon}\left\langle\Omega^{r}\left[x_{0}\right]\right\rangle y, y\right)_{J_{\varepsilon}\langle\Omega\rangle}=\frac{2 \pi}{15} \varepsilon\left(x_{0}\right)\left|\operatorname{curl} y\left(x_{0}\right)\right|^{2}\left(c\left(x_{0}\right) r\right)^{5}+O\left(r^{6}\right), \quad r \rightarrow 0 .
$$

Proof. We assume $x_{0}=0$ and denote $\Omega^{r}[0]$ by $U^{r}$. Let $\tau_{0}(x):=\operatorname{dist}_{c}(x, 0)$. Together with the Euclidean coordinates $x$, we shall use the Riemannian normal coordinates $\widetilde{x}=$ $\left(\widetilde{x}^{1}, \widetilde{x}^{2}, \widetilde{x}^{3}\right)$ related to the origin 0 (see the definition in 14 ). We add a tilde to the notation of the tensors and functions pulled back from coordinates $x$ to coordinates $\widetilde{x}$. When we pass from one set of coordinates to another, the point 0 is mapped to 0 . Since the map $x \mapsto \widetilde{x}$ restricted to $U^{r}$ is smooth in both directions if $r$ is sufficiently small, we have

$$
\widetilde{x}=O(|x|), \quad x=O(|\widetilde{x}|) .
$$

An important property of the coordinates $\widetilde{x}$ is that

$$
|\widetilde{x}|=\widetilde{\tau}_{0}(\widetilde{x}), \quad \tilde{h}^{i j}(0)=\delta^{i j} .
$$

In this proof by $|\cdot|$ we mean the Euclidean length in $\mathbb{R}^{3}$, independently of the metric tensor. We express $\widetilde{h}$ via $h$ in the last identity:

$$
\sum_{m, n} \frac{\partial \widetilde{x}^{i}}{\partial x^{m}} \frac{\partial \widetilde{x}^{j}}{\partial x^{n}} h^{m n}=\delta^{i j} .
$$

Now, using (1.1) and the relation $g^{m n}=\delta^{m n}$, we see that

$$
\frac{\partial \widetilde{x}}{\partial x}\left(\frac{\partial \widetilde{x}}{\partial x}\right)^{t} c^{2}=\mathbb{1}
$$

(by $\partial \widetilde{x} / \partial x$ we denote the Jacobian matrix of the map $x \mapsto \widetilde{x}$ ). Therefore, the matrix $(c \partial \widetilde{x} / \partial x)(0)$ is orthogonal. We may assume that the normal geodesic coordinates are chosen so that this matrix be equal to the identity matrix. It follows that

$$
\widetilde{x}=c^{-1} x+O\left(|x|^{2}\right), \quad \frac{\partial \widetilde{x}}{\partial x}=c^{-1} \mathbb{1}+O(|x|) .
$$

Since $\partial U^{r}$ is a level set of the function $\tau_{0}$, for the normal to this surface we have

$$
n^{i}(x)=n_{i}(x)=\frac{1}{\left|\nabla \tau_{0}\right|} \frac{\partial \tau_{0}}{\partial x^{i}}=c \frac{\partial \tau_{0}}{\partial x^{i}}
$$

(we have used the relation $\left|\nabla \tau_{0}\right|=c^{-1}$ ). Next,

$$
\frac{\partial \tau_{0}}{\partial x^{i}}=\sum_{j} \frac{\partial \widetilde{\tau}_{0}}{\partial \widetilde{x}^{j}} \frac{\partial \widetilde{x}^{j}}{\partial x^{i}}=\sum_{j} \frac{\widetilde{x}^{j}}{|\widetilde{x}|} \frac{\partial \widetilde{x}^{j}}{\partial x^{i}}
$$

(in the second identity we have used the first relation in (8.3)). Now, by (8.4), we obtain

$$
n^{i}(x)=\frac{x^{i}}{|x|}+O(|x|) .
$$

We write an expansion of the field $y$ in the neighborhood of the origin:

$$
y(x)=y(0)+A x+z, \quad A_{i j}:=\frac{\partial y^{i}}{\partial x^{j}}(0), \quad z=O\left(|x|^{2}\right),
$$

and introduce the function $q:=q_{0}+q_{1}$ in $\Omega$, where $q_{0}$ is a function such that $\nabla q_{0}=y(0)$ in $\Omega, q_{1}(x):=\langle A x, x\rangle / 2$. The symmetric and the antisymmetric part of a matrix are defined by:

$$
\operatorname{Re} A:=\left(A+A^{t}\right) / 2, \quad \operatorname{Im} A:=\left(A-A^{t}\right) / 2 .
$$


It is easy to check that

$$
\nabla q_{1}=(\operatorname{Re} A) x
$$

whence

$$
\nabla q=y(0)+(\operatorname{Re} A) x .
$$

Let $p^{r}$ be a solution of problem (8.1) in the domain $U=U^{r}$. For $\varphi \in H^{1}\left(U^{r}\right)$, we have

$$
\begin{aligned}
\left(\varepsilon \nabla\left(p^{r}-q\right), \nabla \varphi\right)_{U^{r}}=(\varepsilon(y-\nabla q), \nabla \varphi)_{U^{r}} & =(\varepsilon(y-y(0)-(\operatorname{Re} A) x), \nabla \varphi)_{U^{r}} \\
& =(\varepsilon(\operatorname{Im} A) x, \nabla \varphi)_{U^{r}}+(\varepsilon z, \nabla \varphi)_{U^{r}}
\end{aligned}
$$

(in this proof by $(\cdot, \cdot)_{X}$ and $\|\cdot\|_{X}$ we denote the inner product and the norm in the space $\vec{L}_{2}(X)$ or $L_{2}(X)$ for a given set $\left.X\right)$. For the second term we have

$$
\left|(\varepsilon z, \nabla \varphi)_{U^{r}}\right| \leq(\varepsilon z, z)_{U^{r}}^{1 / 2}(\varepsilon \nabla \varphi, \nabla \varphi)_{U^{r}}^{1 / 2} \leq C r^{7 / 2}(\varepsilon \nabla \varphi, \nabla \varphi)_{U^{r}}^{1 / 2}
$$

Estimating the first term in (8.9), we get

$$
\begin{aligned}
\varepsilon & =\varepsilon(0)+\varepsilon_{1}, \quad \varepsilon_{1}=O(|x|), \\
(\varepsilon(\operatorname{Im} A) x, \nabla \varphi)_{U^{r}} & =\varepsilon(0)((\operatorname{Im} A) x, \nabla \varphi)_{U^{r}}+\left(\varepsilon_{1}(\operatorname{Im} A) x, \nabla \varphi\right)_{U^{r}} .
\end{aligned}
$$

Here, the second term is estimated as in (8.10):

$$
\left|\left(\varepsilon_{1}(\operatorname{Im} A) x, \nabla \varphi\right)_{U^{r}}\right| \leq C r^{7 / 2}(\varepsilon \nabla \varphi, \nabla \varphi)_{U^{r}}^{1 / 2}
$$

In the first term we change $\varphi$ to a function $\varphi_{0}$ that differs from $\varphi$ by a constant to be specified later. Using the identities

$$
\operatorname{div}((\operatorname{Im} A) x)=\operatorname{Tr} \operatorname{Im} A=0, \quad\langle(\operatorname{Im} A) x, x\rangle=0,
$$

and integrating by parts, we obtain

$$
\begin{aligned}
((\operatorname{Im} A) x, \nabla \varphi)_{U^{r}}=\left((\operatorname{Im} A) x, \nabla \varphi_{0}\right)_{U^{r}} & =\int_{\partial U^{r}}\langle(\operatorname{Im} A) x, n\rangle \varphi_{0} d S_{x} \\
& =\int_{\partial U^{r}}\left\langle(\operatorname{Im} A) x, n-|x|^{-1} x\right\rangle \varphi_{0} d S_{x}
\end{aligned}
$$

Now we estimate the integral obtained:

$$
\left|\int_{\partial U^{r}}\left\langle(\operatorname{Im} A) x, n-|x|^{-1} x\right\rangle \varphi_{0} d S_{x}\right| \leq\left\|\left\langle(\operatorname{Im} A) x, n-|x|^{-1} x\right\rangle\right\|_{\partial U^{r}} \cdot\left\|\varphi_{0}\right\|_{\partial U^{r}}
$$

From (8.5) it follows that $n(x)-|x|^{-1} x=O(|x|)=O(|\widetilde{x}|)$, but if $x \in \partial U^{r}$, then $|\widetilde{x}(x)|=r$ (see $(8.3)$ ), whence

$$
\left.|n(x)-| x\right|^{-1} x \mid \leq C r, \quad x \in \partial U^{r},
$$

where $C$ does not depend on $r$. For a fixed $r$, we put

$$
\widehat{x}(x):=r^{-1} \widetilde{x}(x), \quad \widehat{\varphi}(\widehat{x}):=\varphi(x(\widehat{x})) .
$$

Under the map $x \mapsto \widehat{x}$, the set $U^{r}$ is mapped to the unit ball $B$ in $\mathbb{R}^{3}$ with center at the origin. By (8.14), we have

$$
\left\|\left\langle(\operatorname{Im} A) x, n-|x|^{-1} x\right\rangle\right\|_{\partial U^{r}} \leq C r^{2}\left(\int_{\partial U^{r}} d S\right)^{1 / 2}=C r^{2}\left(\int_{\partial B}\left|\operatorname{det}\left(\frac{\partial S}{\partial \widehat{S}}\right)\right| d \widehat{S}\right)^{1 / 2} .
$$

Direct calculations show that

$$
\left|\operatorname{det}\left(\frac{\partial S}{\partial \widetilde{S}}\right)\right|=c^{-1} \operatorname{det}\left(\frac{\partial x}{\partial \widetilde{x}}\right), \quad\left|\operatorname{det}\left(\frac{\partial \widetilde{S}}{\partial \widehat{S}}\right)\right|=r^{2} .
$$


Therefore,

$$
\left|\operatorname{det}\left(\frac{\partial S}{\partial \widehat{S}}\right)\right|=r^{2} c^{-1} \operatorname{det}\left(\frac{\partial x}{\partial \widetilde{x}}\right) \leq C r^{2},
$$

and thus

$$
\left\|\left\langle(\operatorname{Im} A) x, n-|x|^{-1} x\right\rangle\right\|_{\partial U^{r}}=O\left(r^{3}\right) .
$$

Now we turn to estimation of the second factor in (8.13). Let

$$
\varphi_{0}:=\varphi-(\operatorname{meas} B)^{-1} \int_{B} \hat{\varphi} d \widehat{x}, \quad \hat{\varphi}_{0}:=\widehat{\varphi}-(\text { meas } B)^{-1} \int_{B} \hat{\varphi} d \widehat{x},
$$

Using inequality (8.15), we can estimate the norm of the trace of $\varphi_{0}$ :

$$
\int_{\partial U^{r}}\left|\varphi_{0}\right|^{2} d S=\int_{\partial B}\left|\hat{\varphi}_{0}\right|^{2}\left|\operatorname{det}\left(\frac{\partial S}{\partial \widehat{S}}\right)\right| d \widehat{S} \leq C r^{2} \int_{\partial B}\left|\hat{\varphi}_{0}\right|^{2} d \widehat{S} .
$$

The function $\hat{\varphi}_{0} \in H^{1}(B)$ satisfies the Poincaré inequality

$$
\left\|\hat{\varphi}_{0}\right\|_{B} \leq C\left(\left\|\nabla \hat{\varphi}_{0}\right\|_{B}+\left|\int_{B} \hat{\varphi}_{0} d \hat{x}\right|\right)=C\left\|\nabla \hat{\varphi}_{0}\right\|_{B} .
$$

Together with the trace theorem, this inequality yields the estimate

$$
\left\|\hat{\varphi}_{0}\right\|_{\partial B} \leq \widetilde{C}\left\|\hat{\varphi}_{0}\right\|_{H^{1}(B)} \leq \widehat{C}\left\|\nabla \hat{\varphi}_{0}\right\|_{B}=\widehat{C}\|\nabla \hat{\varphi}\|_{B}
$$

We have

$$
\int_{B}|\nabla \hat{\varphi}|^{2} d \widehat{x}=\int_{U^{r}}\left|\left(\frac{\partial x}{\partial \widehat{x}}\right)^{t} \nabla \varphi\right|^{2} \operatorname{det}\left(\frac{\partial \widehat{x}}{\partial x}\right) d x \leq C r^{-1} \int_{U^{r}}|\nabla \varphi|^{2} d x .
$$

Combined with (8.17) and (8.18), this inequality yields

$$
\left\|\varphi_{0}\right\|_{\partial U^{r}} \leq C r^{1 / 2}\|\nabla \varphi\|_{U^{r}} .
$$

The resulting inequality and relations (8.12), (8.13), and (8.16) show that the first term in (8.11) admits the estimate

$$
\left|((\operatorname{Im} A) x, \nabla \varphi)_{U^{r}}\right| \leq C r^{7 / 2}(\varepsilon \nabla \varphi, \nabla \varphi)_{U^{r}}^{1 / 2} .
$$

Having estimated all terms in (8.9), we collect the results to obtain

$$
\left|\left(\varepsilon \nabla\left(p^{r}-q\right), \nabla \varphi\right)_{U^{r}}\right| \leq \widetilde{C} r^{7 / 2}(\varepsilon \nabla \varphi, \nabla \varphi)_{U^{r}}^{1 / 2} .
$$

Putting $\varphi=p^{r}-q$, we arrive at

$$
\left(\varepsilon \nabla\left(p^{r}-q\right), \nabla\left(p^{r}-q\right)\right)_{U^{r}}=O\left(r^{7}\right) .
$$

Now we turn to the inner product in (8.2):

$$
\begin{aligned}
\left(\varepsilon P_{\varepsilon}\left\langle U^{r}\right\rangle y, y\right)_{U^{r}}= & \left(\varepsilon\left(y-\nabla p^{r}\right), y-\nabla p^{r}\right)_{U^{r}} \\
= & \left(\varepsilon\left(y-\nabla q-\nabla\left(p^{r}-q\right)\right), y-\nabla q-\nabla\left(p^{r}-q\right)\right)_{U^{r}} \\
= & (\varepsilon(y-\nabla q), y-\nabla q)_{U^{r}}-2\left(\varepsilon(y-\nabla q), \nabla\left(p^{r}-q\right)\right)_{U^{r}} \\
& \quad+\left(\varepsilon \nabla\left(p^{r}-q\right), \nabla\left(p^{r}-q\right)\right)_{U^{r}} .
\end{aligned}
$$

The second term of the result is $O\left(r^{6}\right)$. Indeed, from (8.8) it follows that

$$
y-\nabla q=O(|x|), \quad(\varepsilon(y-\nabla q), y-\nabla q)_{U^{r}}=O\left(r^{5}\right),
$$

whence

$$
\left(\varepsilon(y-\nabla q), \nabla\left(p^{r}-q\right)\right)_{U^{r}}=O\left(r^{5 / 2}\right)\left(\varepsilon \nabla\left(p^{r}-q\right), \nabla\left(p^{r}-q\right)\right)_{U^{r}}^{1 / 2}=O\left(r^{6}\right)
$$


(the last estimate follows from (8.19)). The same relation (8.19) also implies that the third term in (8.20) is $O\left(r^{7}\right)$. Now, consider the first term. Using (8.21) and an expansion for $\varepsilon$, we obtain

$$
\begin{aligned}
(\varepsilon(y-\nabla q), y-\nabla q)_{U^{r}} & =\varepsilon(0)(y-\nabla q, y-\nabla q)_{U^{r}}+\left(\varepsilon_{1}(y-\nabla q), y-\nabla q\right)_{U^{r}} \\
& =\varepsilon(0)(y-\nabla q, y-\nabla q)_{U^{r}}+O\left(r^{6}\right) .
\end{aligned}
$$

At this point, we use (8.6) and (8.8) once again:

$$
(y-\nabla q, y-\nabla q)_{U^{r}}=((\operatorname{Im} A) x+z,(\operatorname{Im} A) x+z)_{U^{r}}=((\operatorname{Im} A) x,(\operatorname{Im} A) x)_{U^{r}}+O\left(r^{6}\right) .
$$

For the inner product we have

$$
\begin{aligned}
\int_{U^{r}}|(\operatorname{Im} A) x|^{2} d x & =\int_{|\widetilde{x}|<r}|(\operatorname{Im} A) x(\widetilde{x})|^{2} \operatorname{det}\left(\frac{\partial x}{\partial \widetilde{x}}\right) d \widetilde{x} \\
& =\int_{|\widetilde{x}|<r}\left|(\operatorname{Im} A) \widetilde{c} \widetilde{x}+O\left(|\widetilde{x}|^{2}\right)\right|^{2}\left(\widetilde{c}^{3}+O(|\widetilde{x}|)\right) d \widetilde{x} \\
& =c(0)^{5} \int_{|\widetilde{x}|<r}|(\operatorname{Im} A) \widetilde{x}|^{2} d \widetilde{x}+O\left(r^{6}\right)
\end{aligned}
$$

It remains to calculate the integral in the last expression. Note that

$$
(\operatorname{Im} A) \widetilde{x}=\frac{1}{2} \operatorname{curl} y(0) \times \widetilde{x} .
$$

Put $v:=\operatorname{curl} y(0) / 2$ and denote by $\theta$ the angle between $v$ and $\widetilde{x}$. Now, the following calculation finishes the proof of the lemma:

$$
\begin{aligned}
\int_{|\widetilde{x}|<r}|(\operatorname{Im} A) \widetilde{x}|^{2} d \widetilde{x} & =\int_{|\widetilde{x}|<r}|v \times \widetilde{x}|^{2} d \widetilde{x}=\int_{|\widetilde{x}|<r}|v|^{2}|\widetilde{x}|^{2}(\sin \theta)^{2} d \widetilde{x} \\
& =2 \pi|v|^{2} \int_{0}^{r} d t \int_{0}^{\pi} d \theta t^{4}(\sin \theta)^{3}=2 \pi|v|^{2} \int_{0}^{r} d t t^{4} \frac{4}{3}=\frac{8 \pi}{15}|v|^{2} r^{5} \\
& =\frac{2 \pi}{15}|\operatorname{curl} y(0)|^{2} r^{5} .
\end{aligned}
$$

\section{§. RECOVERING $\varepsilon$ AND $\mu$}

After we have recovered the velocity $c$ in $\Omega^{T}$ and established a correspondence between points of the pattern $\Theta^{T}$ and points of $\Omega^{T}$, we may assume that the operator $\pi$ of parallel transport is known. To prove Theorem 2, it remains to recover $\varepsilon$ in $\Omega^{T}$, because $\mu$ can be derived from (0.2). The function $\varepsilon$ is determined by the gradient $\nabla \ln \varepsilon$ and the value of $\varepsilon$ at any fixed point. But in Subsection 2.2 we noted that the data (2.5) determine $\varepsilon$ on $\Gamma$. To find the gradient $\nabla \ln \varepsilon$, first we find its tangential part $\nabla_{\theta} \ln \varepsilon$ by using the operator $\widetilde{\mathcal{R}}_{e}^{T}$ (defined in Subsection 6.1), and then we find its normal part with the help of the results of $\S \$ 7,8$,

9.1. Recovering $\nabla_{\theta} \ln \varepsilon$. Here we show that $\nabla_{\theta} \ln \varepsilon$ can be recovered in $\Omega^{T}$ by using $\widetilde{\mathcal{R}}_{e}^{T}$. Let $\overrightarrow{\mathcal{L}}_{2}\left(\Omega^{T}\right)$ denote the space $\overrightarrow{\mathcal{L}}_{2, \eta}\left(\Omega^{T}\right)$ (defined in Subsection 4.2 ) for $\eta=1$. We pass from $\widetilde{\mathcal{R}}_{e}^{T}$ to the operator

$$
\pi^{-1} \widetilde{\mathcal{R}}_{e}^{T} \pi-c \Pi \text { curl }: \overrightarrow{\mathcal{L}}_{2}\left(\Omega^{T}\right) \rightarrow \overrightarrow{\mathcal{L}}_{2}\left(\Omega^{T}\right)
$$


defined in $\vec{C}_{0}^{\infty}\left(\Omega^{T} \backslash \omega\right) \cap \overrightarrow{\mathcal{L}}_{2}\left(\Omega^{T}\right)$. In accordance with (6.3), the operator (9.1) acts on a field $u$ as follows:

$$
\begin{aligned}
\left.\left(\pi^{-1} \widetilde{\mathcal{R}}_{e}^{T} \pi u-c \Pi \operatorname{curl} u\right)\right|_{\Gamma^{s}}=[ & \left.\varkappa_{\mu} \Pi \mu^{-1} \operatorname{curl}\left(\varkappa_{\varepsilon}^{-1} u\right)-c \Pi \operatorname{curl} u\right]\left.\right|_{\Gamma^{s}} \\
& -\varkappa_{\mu} c^{-1} \mu^{-1} N \nabla_{\theta} \Lambda_{\varepsilon}^{s}\left(\varepsilon^{-1} \operatorname{div}_{\theta}\left(c \varepsilon \varkappa_{\varepsilon}^{-1} u\right)\right) \\
& +\varkappa_{\mu} \nabla_{\theta} \Lambda_{\mu}^{s}\left(\mu^{-1} \operatorname{div}_{\theta}\left(N \varkappa_{\varepsilon}^{-1} u\right)\right)+\left.A_{00} u\right|_{\Gamma^{s}} .
\end{aligned}
$$

We have denoted by $A_{00}$ the operator corresponding to the terms with projections $B^{s}$ in (6.3). In the proof of Lemma 4 it was shown that this operator is fiberwise; the corresponding operators acting on equidistant surfaces are denoted by $A_{00}(s)$.

Since $\varkappa_{\mu} \mu^{-1} \varkappa_{\varepsilon}^{-1}=c$, we have

$$
\varkappa_{\mu} \Pi \mu^{-1} \operatorname{curl} \varkappa_{\varepsilon}^{-1} u-c \Pi \operatorname{curl} u=\varkappa_{\mu} \mu^{-1} \Pi\left(\nabla \varkappa_{\varepsilon}^{-1} \times u\right)=\varkappa_{\mu} \mu^{-1}\left\langle\nabla \varkappa_{\varepsilon}^{-1}, \nu\right\rangle N u .
$$

It follows that the right-hand side in (9.2) acts on $u$ as a fiberwise operator. The factor in front of $N u$ in the above expression is denoted by $\zeta$. By (4.8) and (0.2), we have

$$
\varkappa_{\mu}=\varkappa c^{-1} \varepsilon^{-1 / 2} \text {. }
$$

Fixing $s$, we consider the composition

$$
\operatorname{div}_{\theta}\left(\pi^{-1} \tilde{\mathcal{R}}_{e}^{T} \pi-c \Pi \operatorname{curl}\right) \nabla_{\theta}: C_{0}^{\infty}\left(\Gamma^{s} \backslash \omega\right) \rightarrow C^{\infty}\left(\Gamma^{s} \backslash \omega\right) .
$$

Using (9.3) and the formulas

$$
\operatorname{div}_{\theta}(f w)=\left\langle\nabla_{\theta} f, w\right\rangle+f \operatorname{div}_{\theta} w, \quad \operatorname{div}_{\theta}\left(N \nabla_{\theta} f\right)=0
$$

for $f \in C^{\infty}\left(\Gamma^{s} \backslash \omega\right)$ and a smooth tangential field $w$ on $\Gamma^{s} \backslash \omega$, we can write the action of (9.4) on $\varphi \in C_{0}^{\infty}\left(\Gamma^{s} \backslash \omega\right)$ as follows:

$$
\begin{aligned}
\operatorname{div}_{\theta}\left(\pi^{-1}\right. & \left.\widetilde{\mathcal{R}}_{e}^{T} \pi-c \Pi \text { curl }\right) \nabla_{\theta} \varphi \\
=\langle & \left\langle\nabla_{\theta} \zeta, N \nabla_{\theta} \varphi\right\rangle-\left\langle\nabla_{\theta} \varkappa_{\varepsilon}, N \nabla_{\theta} \Lambda_{\varepsilon}^{s}\left(\varepsilon^{-1} \operatorname{div}_{\theta}\left(c \varepsilon^{1 / 2} \varkappa^{-1} \nabla_{\theta} \varphi\right)\right)\right\rangle \\
& \quad+\operatorname{div}_{\theta}\left(\varkappa c^{-1} \varepsilon^{-1 / 2} \nabla_{\theta} \Lambda_{\mu}^{s}\left(\varepsilon c^{2}\left\langle\nabla_{\theta} \varkappa_{\varepsilon}^{-1}, N \nabla_{\theta} \varphi\right\rangle\right)\right)+\operatorname{div}_{\theta}\left(A_{00}(s) \nabla_{\theta} \varphi\right) .
\end{aligned}
$$

The operator $A_{00}(s)$ is smoothing (see the proof of Lemma 4), the same refers to the last term in the sum; the first term is a first order differential operator. The entire sum is a second order $\psi$.d.o. We fix local coordinates $\left(\gamma^{1}, \gamma^{2}\right)$ on $\Gamma^{s}$. In what follows, we shall use formulas for the principal parts of the symbols of $\operatorname{div}_{\theta}, \nabla_{\theta}$, and $N$, as well as the notation $G$ and $|k|_{g}\left(k \in \mathbb{R}^{2}\right)$ occurring in the proof of Lemma 5. We calculate the principal part of the symbol of the second term in (9.6):

$$
\begin{aligned}
& \frac{-c}{\varkappa \varepsilon^{1 / 2}|k|_{g}}\left(\partial_{1} \varkappa_{\varepsilon}, \partial_{2} \varkappa_{\varepsilon}\right) \frac{1}{\sqrt{\operatorname{det} G}}\left(\begin{array}{cc}
0 & -1 \\
1 & 0
\end{array}\right)\left(\begin{array}{l}
i k_{1} \\
i k_{2}
\end{array}\right)\left(i k_{1}, i k_{2}\right) G^{-1}\left(\begin{array}{l}
i k_{1} \\
i k_{2}
\end{array}\right) \\
& =\frac{c|k|_{g}}{\sqrt{\operatorname{det} G}}\left(\partial_{1} \ln \varkappa_{\varepsilon}, \partial_{2} \ln \varkappa_{\varepsilon}\right)\left(\begin{array}{c}
c-i k_{2} \\
i k_{1}
\end{array}\right)=\frac{c|k|_{g}}{\sqrt{\operatorname{det} G}} i\left(-k_{2} \partial_{1} \ln \varkappa_{\varepsilon}+k_{1} \partial_{2} \ln \varkappa_{\varepsilon}\right),
\end{aligned}
$$

where $\partial_{j}:=\partial / \partial \gamma^{j}$. For the principal part of the symbol of the third term, we have

$$
\begin{aligned}
& \frac{c \varkappa \varepsilon^{1 / 2}}{|k|_{g}}\left(i k_{1}, i k_{2}\right) G^{-1}\left(\begin{array}{l}
i k_{1} \\
i k_{2}
\end{array}\right)\left(\partial_{1} \varkappa_{\varepsilon}^{-1}, \partial_{2} \varkappa_{\varepsilon}^{-1}\right) \frac{1}{\sqrt{\operatorname{det} G}}\left(\begin{array}{cc}
0 & -1 \\
1 & 0
\end{array}\right)\left(\begin{array}{l}
i k_{1} \\
i k_{2}
\end{array}\right) \\
& =-\frac{c|k|_{g}}{\sqrt{\operatorname{det} G}}\left(\partial_{1} \ln \varkappa_{\varepsilon}^{-1}, \partial_{2} \ln \varkappa_{\varepsilon}^{-1}\right)\left(\begin{array}{c}
-i k_{2} \\
i k_{1}
\end{array}\right) \\
& =-\frac{c|k|_{g}}{\sqrt{\operatorname{det} G}} i\left(-k_{2} \partial_{1} \ln \varkappa_{\varepsilon}^{-1}+k_{1} \partial_{2} \ln \varkappa_{\varepsilon}^{-1}\right) \text {. }
\end{aligned}
$$


Thus, for the principal part of the symbol of the operator (9.4) we have

$$
a\left(s ; \gamma^{1}, \gamma^{2}, k\right)=\frac{2 c|k|_{g}}{\sqrt{\operatorname{det} G}} i\left(-k_{2} \partial_{1} \ln \varkappa_{\varepsilon}+k_{1} \partial_{2} \ln \varkappa_{\varepsilon}\right) .
$$

Although (9.4) seems to be a third order operator, our calculations show that in fact it is a second order operator.

Clearly, we can find $\nabla_{\theta} \ln \varkappa_{\varepsilon}$ at $x\left(\gamma\left(\gamma^{1}, \gamma^{2}\right), s\right)$ if we know the values of $a\left(s ; \gamma^{1}, \gamma^{2}, k\right)$ for different $k$, and then $\nabla_{\theta} \ln \varepsilon$ can be found, because $\ln \varkappa_{\varepsilon}=\ln \varkappa+(\ln \varepsilon) / 2$ and the function $\varkappa$ is known once we have recovered $c$ in $\Omega^{T}$.

9.2. The action of $\left(M^{T}\right)^{*}$ on transversal solenoidal fields. To find $\langle\nabla \ln \varepsilon, \nu\rangle$, we need the following lemma.

Lemma 8. Let $Z$ be a closed subset of $\Gamma$ homeomorphic to a disk, and let $s_{0} \in(0, T)$ be a number such that $Z \times\left(0, s_{0}\right] \subset \Theta^{T}$. Suppose a smooth field $u \in J_{\varepsilon}^{T} \cap \overrightarrow{\mathcal{L}}_{2}\left(\Omega^{T}\right)$ satisfies $\operatorname{supp} u \subset V, V:=i^{-1}\left(Z \times\left(0, s_{0}\right)\right)$. Then $u \in \mathcal{U}_{\varepsilon}^{T}$ and

$$
M_{\varepsilon}^{T} u=\left(M_{\varepsilon}^{T}\right)^{*} u=u .
$$

Proof. The inclusion $u \in \mathcal{U}_{\varepsilon}^{T}$ follows from (7.3). To prove (9.7), we establish the relation

$$
B_{\varepsilon}^{s} u=0
$$

for all $s \in(0, T]$. Let $\alpha \in J_{\varepsilon}^{s} \ominus \mathcal{U}_{\varepsilon}^{s}$. Choose an open set $U^{s} \subset V \cap \Omega^{s}$ such that $\operatorname{supp} u \cap \Omega^{s} \subset U^{s}, \partial U^{s} \in C^{\infty}$. Clearly, the set $V \cap \Omega^{s}$ is homeomorphic to a ball; therefore, the restriction of $\alpha$ to $U^{s}$ equals $\nabla \varphi, \varphi \in C^{\infty}\left(\overline{U^{s}}\right)$. Let $n$ be an outward normal on the boundary $\partial U^{s}$. Then $\langle u, n\rangle$ vanishes on the boundary, because $n$ coincides with $\nu$ on $\partial U^{s} \cap \Gamma^{s}$ and $u$ is transversal, while on the remaining part $\partial U^{s} \backslash \Gamma^{s}$ of the boundary the function $u$ vanishes. Therefore,

Hence,

$$
(u, \alpha)_{\vec{L}_{2, \varepsilon}\left(\Omega^{s}\right)}=\int_{U^{s}}\langle\varepsilon u, \nabla \varphi\rangle d x=\int_{\partial U^{s}} \varepsilon\langle u, n\rangle \varphi d S=0
$$

which proves (9.8).

$$
u \perp J_{\varepsilon}^{s} \ominus \mathcal{U}_{\varepsilon}^{s},
$$

Since the field $u$ is transversal, formulas (4.4) and (9.8) show that $E_{\varepsilon}^{s} u$ coincides with $u$ on $\Omega^{s}$ for all $s \in(0, T]$. Recalling the definition (4.7), we see that $M_{\varepsilon}^{T} u=u$. By item $(b)$ of Theorem 3, the operator $M_{\varepsilon}^{T}$ is partially isometric, and the identity proved above implies that $u \perp \operatorname{Ker} M_{\varepsilon}^{T}$. Then $u \in \operatorname{Ran}\left(M_{\varepsilon}^{T}\right)^{*}$, i.e., for some $\widetilde{u} \in \overrightarrow{\mathcal{L}}_{2}\left(\Omega^{T}\right)$ we have

$$
u=\left(M_{\varepsilon}^{T}\right)^{*} \widetilde{u} .
$$

Now we apply item $(b)$ of Theorem 3 once again:

$$
u=M_{\varepsilon}^{T} u=M_{\varepsilon}^{T}\left(M_{\varepsilon}^{T}\right)^{*} \widetilde{u}=\widetilde{u} .
$$

Consequently, $\left(M_{\varepsilon}^{T}\right)^{*} u=u$.

9.3. Recovering $\langle\nabla \ln \varepsilon, \nu\rangle$. We fix a point $x_{0} \in \Omega^{T} \backslash \omega$ and an open set $U \subset \Omega^{T} \backslash \omega$ containing $x_{0}$. Suppose that $f$ is a positive function in $U$ satisfying

$$
\nabla_{\theta} \ln f=-\nabla_{\theta} \ln \left(\varepsilon \varkappa_{\varepsilon}^{-1}\right),
$$

where the right-hand side is determined by $c$ and $\nabla_{\theta} \ln \varepsilon$. For an arbitrary $\varphi \in C_{0}^{\infty}(U)$, the transversal field

satisfies $\operatorname{div}\left(\varepsilon \varkappa_{\varepsilon}^{-1} u\right)=0$. Indeed,

$$
u:=f c^{-1} N \nabla_{\theta} \varphi
$$

$$
\operatorname{div}\left(\varepsilon \varkappa_{\varepsilon}^{-1} f c^{-1} N \nabla_{\theta} \varphi\right)=c^{-1} \operatorname{div}_{\theta}\left(\varepsilon \varkappa_{\varepsilon}^{-1} f N \nabla_{\theta} \varphi\right)=c^{-1}\left\langle\nabla_{\theta}\left(\varepsilon \varkappa_{\varepsilon}^{-1} f\right), N \nabla_{\theta} \varphi\right\rangle
$$


(we have applied (6.2) and (9.5)), but

$$
\frac{\nabla_{\theta}\left(\varepsilon \varkappa_{\varepsilon}^{-1} f\right)}{\varepsilon \varkappa_{\varepsilon}^{-1} f}=\nabla_{\theta} \ln f+\nabla_{\theta} \ln \left(\varepsilon \varkappa_{\varepsilon}^{-1}\right)=0,
$$

whence $\nabla_{\theta}\left(\varepsilon \varkappa_{\varepsilon}^{-1} f\right)=0$. We may assume that the function $\varphi$ is such that $u\left(x_{0}\right) \neq 0$ and

$$
\beta u\left(x_{0}\right)=\nabla_{\theta} \ln \varepsilon
$$

for some $\beta \in \mathbb{R}$. Next, we introduce the family

$$
u_{(n)}(x):=e^{n\left(\tau(x)-\tau\left(x_{0}\right)\right)} u(x),
$$

of transversal fields, parametrized by real numbers $n$. It is easily seen that

$$
\operatorname{div}\left(\varepsilon \varkappa_{\varepsilon}^{-1} u_{(n)}\right)=0
$$

for all $n$. Let

$$
y_{(n)}:=\left(I_{\varepsilon}^{T}\right)^{*} \pi u_{(n)}=\left(M_{\varepsilon}^{T}\right)^{*} \varkappa_{\varepsilon}^{-1} u_{(n)}
$$

(here we have used (4.10) ). If $U$ is a sufficiently small neighborhood of the point $x_{0}$, then the fields $\varkappa_{\varepsilon}^{-1} u_{(n)}$ satisfy the conditions of Lemma 8 , hence,

$$
y_{(n)}=\varkappa_{\varepsilon}^{-1} u_{(n)}=\varepsilon^{-1 / 2} \varkappa^{-1} u_{(n)} .
$$

In the sequel, we deal with the fields $v_{(n)}:=\varkappa^{-1} u_{(n)}$. The above identity implies that

$$
\operatorname{curl} y_{(n)}=\nabla \varepsilon^{-1 / 2} \times v_{(n)}+\varepsilon^{-1 / 2} \operatorname{curl} v_{(n)} .
$$

We put $b:=\left\langle\nabla \ln \varepsilon^{-1 / 2}, \nu\right\rangle$ and calculate the first term at $x_{0}$ :

$$
\begin{aligned}
\nabla \varepsilon^{-1 / 2} \times v_{(n)} & =\varepsilon^{-1 / 2} \nabla \ln \varepsilon^{-1 / 2} \times v_{(n)} \\
& =\varepsilon^{-1 / 2}\left(\nabla_{\theta} \ln \varepsilon^{-1 / 2} \times v_{(n)}+b \nu \times v_{(n)}\right)=\varepsilon^{-1 / 2} b N v_{(n)}
\end{aligned}
$$

(we have used (9.9)). Next,

$$
\begin{aligned}
\left(\varepsilon\left|\operatorname{curl} y_{(n)}\right|^{2}\right)\left(x_{0}\right) & =\left(\left|b N v_{(n)}+\operatorname{curl} v_{(n)}\right|^{2}\right)\left(x_{0}\right) \\
& =\left(\left|b v_{(n)}\right|^{2}+2 b\left\langle N v_{(n)}, \operatorname{curl} v_{(n)}\right\rangle+\left|\operatorname{curl} v_{(n)}\right|^{2}\right)\left(x_{0}\right) .
\end{aligned}
$$

For the second term we have

$$
\begin{aligned}
\left\langle N v_{(n)}, \operatorname{curl} v_{(n)}\right\rangle & =\left\langle N v_{(n)}, \nabla e^{n\left(\tau(x)-\tau\left(x_{0}\right)\right)} \times v_{(0)}+e^{n\left(\tau(x)-\tau\left(x_{0}\right)\right)} \operatorname{curl} v_{(0)}\right\rangle \\
& =\left\langle N v_{(n)}, n e^{n\left(\tau(x)-\tau\left(x_{0}\right)\right)} \nabla \tau \times v_{(0)}+e^{n\left(\tau(x)-\tau\left(x_{0}\right)\right)} \operatorname{curl} v_{(0)}\right\rangle \\
& =\left\langle v_{(n)}, n e^{n\left(\tau(x)-\tau\left(x_{0}\right)\right)} c^{-1} v_{(0)}\right\rangle+\left\langle N v_{(n)}, e^{n\left(\tau(x)-\tau\left(x_{0}\right)\right)} \operatorname{curl} v_{(0)}\right\rangle
\end{aligned}
$$

(at the end we have used the formula $\nabla \tau=c^{-1} \nu,|\nu|=1$ ).

The value of the first term in (9.10) at $x_{0}$ equals $\left|b v_{(0)}\right|^{2}$ (it does not depend on $n$ ). The previous calculation shows that the second term at $x_{0}$ is equal to $n \cdot 2 b c^{-1}\left|v_{(0)}\right|^{2}+$ $2 b\left\langle N v_{(0)}, \operatorname{curl} v_{(0)}\right\rangle$. Thus,

$$
\begin{aligned}
& \left(\varepsilon\left|\operatorname{curl} y_{(n)}\right|^{2}-\left|\operatorname{curl} v_{(n)}\right|^{2}\right)\left(x_{0}\right) \\
& \quad=n \cdot\left(2 b c^{-1}\left|v_{(0)}\right|^{2}\right)\left(x_{0}\right)+\left(2 b\left\langle N v_{(0)}, \operatorname{curl} v_{(0)}\right\rangle+\left|b v_{(0)}\right|^{2}\right)\left(x_{0}\right) .
\end{aligned}
$$

Before applying (8.2) to the field $y_{(n)}$, observe that for the projections $P_{\varepsilon \#}\left\langle\Omega^{r}\left[x_{0}\right]\right\rangle$ onto the spaces $J_{\varepsilon \#}\left\langle\Omega^{r}\left[x_{0}\right]\right\rangle$ acting in $\mathcal{U}_{\varepsilon \#}^{T}$ we have

$$
\left(P_{\varepsilon}\left\langle\Omega^{r}\left[x_{0}\right]\right\rangle y_{(n)}, y_{(n)}\right)_{J_{\varepsilon}^{T}}=\left(P_{\varepsilon \#}\left\langle\Omega^{r}\left[x_{0}\right]\right\rangle y_{(n) \#}, y_{(n) \#}\right)_{\mathcal{U}_{\varepsilon \#}^{T}},
$$

where

$$
y_{(n) \#}:=\left(\Phi^{T}\right)^{*} y_{(n)}
$$


Combined with (8.2), this implies

$$
\left(\varepsilon\left|\operatorname{curl} y_{(n)}\right|^{2}\right)\left(x_{0}\right)=\frac{15}{2 \pi} \lim _{r \rightarrow 0} \frac{\left(P_{\varepsilon \#}\left\langle\Omega^{r}\left[x_{0}\right]\right\rangle y_{(n) \#}, y_{(n) \#}\right)_{\mathcal{U}_{\varepsilon \#}^{T}}}{\left(c\left(x_{0}\right) r\right)^{5}} .
$$

By the definition of $y_{(n)}$, the elements $y_{(n) \#}$ can be expressed via $u_{(n)}$ in the following way:

$$
y_{(n) \#}=\left(I_{\varepsilon \#}^{T}\right)^{*} \pi u_{(n)} .
$$

Thus, with the help of the model image operator and the model projections we can find $\left(\varepsilon\left|\operatorname{curl} y_{(n)}\right|^{2}\right)\left(x_{0}\right)$, whence we can find the left-hand side of (9.11) for any $n$. The righthand side is a first degree polynomial in $n$. To find its coefficients, it suffices to know the values of the left-hand side for two different $n$. In its turn, $b$ can be expressed via the coefficient of $n$ in this polynomial, because $v_{(0)}\left(x_{0}\right) \neq 0$.

\section{$\S 10$. Appendix}

Here we prove Lemma 2. We follow the lines of the proof of a similar result for the scalar wave equation, as given in 4. In this section we assume that all function spaces are complex. The parentheses $\langle\cdot, \cdot\rangle$ denote the standard inner product in $\mathbb{C}^{3}$.

10.1. The operator $\left(W^{T}\right)^{*}$. Consider the following initial boundary-value problem for the Maxwell system in $\Omega \times \mathbb{R}$ :

$$
\begin{aligned}
& E_{t}=\varepsilon^{-1} \operatorname{curl} H, \quad H_{t}=-\mu^{-1} \operatorname{curl} E, \\
& \left.E\right|_{t=T}=y,\left.\quad H\right|_{t=T}=z, \\
& \left.E_{\theta}\right|_{\Gamma}=0
\end{aligned}
$$

To describe solutions of this problem for nonsmooth initial data, we introduce the subspaces

$$
\begin{aligned}
& J_{\theta}:=\left\{y \in \vec{L}_{2, \varepsilon}(\Omega) \mid \operatorname{div}(\varepsilon y)=0\right\} \subset \vec{L}_{2, \varepsilon}(\Omega), \\
& J_{\nu}:=\cos _{\vec{L}_{2, \mu}}\left\{z \in \vec{C}_{0}^{\infty}(\Omega) \mid \operatorname{div}(\mu z)=0\right\} \subset \vec{L}_{2, \mu}(\Omega),
\end{aligned}
$$

and an operator $\mathcal{R}_{\theta}: J_{\theta} \rightarrow J_{\nu}$ :

$$
\mathcal{R}_{\theta} y:=\mu^{-1} \operatorname{curl} y, \quad \operatorname{Dom} \mathcal{R}_{\theta}:=\left\{y \in J_{\theta}\left|\operatorname{curl} y \in \vec{L}_{2}(\Omega), y_{\theta}\right|_{\Gamma}=0\right\} .
$$

It can be shown (see [15], 9]) that the operator $\mathcal{R}_{\theta}$ is densely defined in $J_{\theta}$ and is closed, $\operatorname{Ran} \mathcal{R}_{\theta} \subset J_{\nu}$, and that the adjoint operator $\mathcal{R}_{\nu}:=\left(\mathcal{R}_{\theta}\right)^{*}$ looks like this:

$$
\mathcal{R}_{\nu} z=\varepsilon^{-1} \operatorname{curl} z, \quad \operatorname{Dom} \mathcal{R}_{\nu}=\left\{z \in J_{\nu} \mid \operatorname{curl} z \in \vec{L}_{2}(\Omega)\right\} .
$$

Clearly, $\mathcal{U}_{\varepsilon}^{T} \subset J_{\theta}$. The definition of the space $\mathcal{U}_{\varepsilon}^{T}$ implies that

$$
\mathcal{U}_{\varepsilon}^{T} \perp \operatorname{Ker} \mathcal{R}_{\theta} .
$$

Indeed, let $w \in \operatorname{Dom} \mathcal{R}_{\theta}$. For an arbitrary element $y \in \mathcal{U}_{\varepsilon}^{T}$ such that $y=\varepsilon^{-1} \operatorname{curl} z$, $z \in \vec{C}^{\infty}(\bar{\Omega})$, supp $z \subset \Omega^{T} \cup \Gamma$, we have

$$
(y, w)_{\vec{L}_{2, \varepsilon}(\Omega)}=(\operatorname{curl} z, w)_{\vec{L}_{2, \varepsilon}(\Omega)}=(z, \operatorname{curl} w)_{\vec{L}_{2, \varepsilon}(\Omega)} .
$$

Here we have used the definition (3.5) of the condition $\left.w_{\theta}\right|_{\partial \Omega}=0$. So, for any $w \in \operatorname{Ker} \mathcal{R}_{\theta}$ we have $y \perp w$, whence $y \perp \operatorname{Ker} \mathcal{R}_{\theta}$. Since this is true for a dense subset in $\mathcal{U}_{\varepsilon}^{T}$ (see the definition (2.1)), relation (10.2) is proved.

With the help of the antiselfadjoint Maxwell operator

$$
\mathcal{M}:=\left(\begin{array}{cc}
0 & \mathcal{R}_{\nu} \\
-\mathcal{R}_{\theta} & 0
\end{array}\right)
$$


acting in the space $J_{\theta} \oplus J_{\nu}$, we can write the solution of problem (10.1) with the initial data $y \in J_{\theta}, z \in J_{\nu}$ as follows:

$$
\left(\begin{array}{l}
E(t) \\
H(t)
\end{array}\right)=e^{(t-T) \mathcal{M}}\left(\begin{array}{l}
y \\
z
\end{array}\right) .
$$

Note that if $\{y, z\} \in \operatorname{Dom} \mathcal{M}$, then $\{E(\cdot, t), H(\cdot, t)\} \in \operatorname{Dom} \mathcal{M}$ for all $t$. In this case the fields $E(\cdot, t), H(\cdot, t)$ belong to $\vec{H}^{1}(\Omega)$, because $\Omega$ is a domain with smooth boundary, so that the traces of these fields are well defined on $\Gamma$.

The following lemma describes the operator $\left(W^{T}\right)^{*}$.

Lemma 9. For $f \in \mathcal{F}_{0}^{T}$ and $y \in \operatorname{Dom} \mathcal{R}_{\theta}$, we have

$$
\left(W^{T} f, y\right)_{J_{\theta}}=-\int_{0}^{T} d t \int_{\Gamma} d \Gamma_{x}\langle f(x, t), N H(x, t)\rangle,
$$

where $H$ is the magnetic part of the solution $\{E, H\}$ of problem (10.1) with the initial data

$$
\left.E\right|_{t=T}=y,\left.\quad H\right|_{t=T}=0 .
$$

Proof. Taking the initial conditions of problem (0.1) into account, we get

$$
\int_{0}^{T} d t\left[(e(\cdot, t), E(\cdot, t))_{J_{\theta}}+(h(\cdot, t), H(\cdot, t))_{J_{\nu}}\right]_{t}=(e(\cdot, T), E(\cdot, T))_{J_{\theta}}=\left(W^{T} f, y\right)_{J_{\theta}} .
$$

On the other hand, since $\{E(\cdot, t), H(\cdot, t)\} \in \operatorname{Dom} \mathcal{M}$, the Maxwell equations allow us to transform the integrand as follows:

$$
\begin{aligned}
& {\left[(e(\cdot, t), E(\cdot, t))_{J_{\theta}}+(h(\cdot, t), H(\cdot, t))_{J_{\nu}}\right]_{t}} \\
& \quad=(\operatorname{curl} h(\cdot, t), E(\cdot, t))_{\vec{L}_{2}(\Omega)}-(h(\cdot, t), \operatorname{curl} E(\cdot, t))_{\vec{L}_{2}(\Omega)} \\
& \quad+(e(\cdot, t), \operatorname{curl} H(\cdot, t))_{\vec{L}_{2}(\Omega)}-(\operatorname{curl} e(\cdot, t), H(\cdot, t))_{\vec{L}_{2}(\Omega)} .
\end{aligned}
$$

Since $E(\cdot, t) \in \operatorname{Dom} \mathcal{R}_{\theta}$, we have $\left.E_{\theta}(\cdot, t)\right|_{\Gamma}=0$, and the sum of the first two terms vanishes. For the remaining terms we have

$$
\begin{aligned}
& (e(\cdot, t), \operatorname{curl} H(\cdot, t))_{\vec{L}_{2}(\Omega)}-(\operatorname{curl} e(\cdot, t), H(\cdot, t))_{\vec{L}_{2}(\Omega)} \\
& \quad=-\int_{\Gamma}\langle e(x, t), N H(x, t)\rangle d \Gamma_{x}=-\int_{\Gamma}\langle f(x, t), N H(x, t)\rangle d \Gamma_{x} .
\end{aligned}
$$

Combining this with (10.5), we arrive at (10.4).

10.2. Smoothing. We fix an even function $\varphi \in C_{0}^{\infty}(\mathbb{R})$ such that $\varphi \geq 0$, supp $\varphi \subset$ $[-1 ; 1], \int_{\mathbb{R}} \varphi(t) d t=1$, and for $\delta>0$ set

$$
\varphi^{\delta}(t):=\delta^{-1} \varphi\left(\delta^{-1} t\right), \quad t \in \mathbb{R} .
$$

Also, we introduce the following function on $\mathbb{R}$ :

$$
\rho^{\delta}(\lambda):=\int_{-\delta}^{\delta} \varphi^{\delta}(\eta) e^{-i \eta \lambda} d \eta
$$

and the smoothing operator $G^{\delta}:=\rho^{\delta}(i \mathcal{M})$ acting on the space $J_{\theta} \oplus J_{\nu}$. Since the function $\rho^{\delta}$ is real and bounded, $G^{\delta}$ is a bounded selfadjoint operator. Moreover, at infinity $\rho^{\delta}$ decays faster than any power of $\lambda$; in particular, this implies that

$$
\operatorname{Ran} G^{\delta} \subset \operatorname{Dom} \mathcal{M}, \quad G^{\delta} \mathcal{M}=\mathcal{M} G^{\delta}
$$

(the second relation holds true on Dom $\mathcal{M}$ ). On the other hand, the limit

$$
\lim _{\delta \rightarrow 0} \rho^{\delta}(\lambda)=1
$$


is attained uniformly with respect to $\lambda \in I$ for any interval $I \subset \mathbb{R}$, and, therefore, the sequence $G^{\delta}$ converges strongly to the identity as $\delta \rightarrow 0$.

Next, we show that the matrix representing $G^{\delta}$ in the decomposition $J_{\theta} \oplus J_{\nu}$ is diagonal:

$$
G^{\delta}=\left(\begin{array}{cc}
G_{e}^{\delta} & 0 \\
0 & G_{m}^{\delta}
\end{array}\right)
$$

Consider the unitary operator $S:=\mathbb{1} \oplus(-\mathbb{1})$ in $J_{\theta} \oplus J_{\nu}$. Since $\mathcal{M}$ and $-\mathcal{M}$ are unitarily equivalent, $S \mathcal{M} S^{-1}=-\mathcal{M}$, the spectral measure $\mathcal{P}$ of the operator $i \mathcal{M}$ is unitarily equivalent to the spectral measure $\mathcal{P}_{-}$of the operator $-i \mathcal{M}$. Hence for any Borel set $\mathcal{B} \subset \mathbb{R}$ we have

$$
S \mathcal{P}(\mathcal{B}) S^{-1}=\mathcal{P}_{-}(\mathcal{B})=\mathcal{P}(-\mathcal{B})
$$

Furthermore, since the function $\rho^{\delta}$ is even and $\rho^{\delta}(0)=1$, we can write

$$
G^{\delta}=\mathcal{P}(\{0\})+\int_{(0, \infty)} \rho^{\delta}(\lambda) d \mathcal{P}_{\lambda}+S\left[\int_{(0, \infty)} \rho^{\delta}(\lambda) d \mathcal{P}_{\lambda}\right] S^{-1} .
$$

Obviously, $S^{-1}=S$, so that the sum of the second and the third term commutes with $S$. The same is true for the first term by (10.8). Thus, $\left[G^{\delta}, S\right]=0$, which implies (10.7).

By (10.7), relations (10.6) imply that

(10.9) $\operatorname{Ran} G_{e}^{\delta} \subset \operatorname{Dom} \mathcal{R}_{\theta}, \quad \operatorname{Ran} G_{m}^{\delta} \subset \operatorname{Dom} \mathcal{R}_{\nu}, \quad G_{m}^{\delta} \mathcal{R}_{\theta}=\mathcal{R}_{\theta} G_{e}^{\delta}, \quad G_{e}^{\delta} \mathcal{R}_{\nu}=\mathcal{R}_{\nu} G_{m}^{\delta}$

(the identities are valid on Dom $\mathcal{R}_{\theta}$ and $\operatorname{Dom} \mathcal{R}_{\nu}$, respectively).

For $f \in \mathcal{F}_{00}^{T}$, we introduce the following procedure of smoothing $f \mapsto f^{\delta}, \delta>0$ :

$$
\tilde{f}(t):=\left\{\begin{array}{ll}
-f(2 T-t) & \text { if } \quad T<t \leq 2 T, \\
f(t) & \text { if } \quad 0 \leq t \leq T, \\
0 & \text { if } t<0 \vee t>2 T,
\end{array} \quad f^{\delta}:=\varphi^{\delta} * \tilde{f} .\right.
$$

We assume that the functions $\tilde{f}$ and $f^{\delta}$ are defined for all $t \in \mathbb{R}$.

By analogy with the space $\mathcal{F}_{0}^{T, s}$ of delayed controls, we introduce yet another space

$$
\mathcal{F}_{00}^{T, s}:=\left\{f \in \mathcal{F}_{00}^{T} \mid \operatorname{supp} f \subset \Gamma \times(T-s, T]\right\} .
$$

Lemma 10. Suppose $f \in \mathcal{F}_{00}^{T, s}$ and $\delta \in(0, T-s)$. Then

$$
W^{T} f^{\delta}=G_{e}^{\delta} W^{T} f
$$

Proof. We shall prove the equivalent claim:

$$
\left(W^{T} f^{\delta}, y\right)_{J_{\theta}}=\left(G_{e}^{\delta} W^{T} f, y\right)_{J_{\theta}}, \quad y \in \operatorname{Dom} R_{\theta} .
$$

The solution $\left\{E^{\delta}, H^{\delta}\right\}$ of problem (10.1) with the initial data $\left\{G_{e}^{\delta} y, 0\right\}$ and the solution $\{E, H\}$ of problem (10.1) with the data $\{y, 0\}$ are related to each other as follows:

$$
E^{\delta}=\varphi^{\delta} * E, \quad H^{\delta}=\varphi^{\delta} * H .
$$

This is a consequence of the following calculation, where we use (10.3):

$$
\begin{aligned}
\left(\begin{array}{c}
\varphi^{\delta} * E \\
\varphi^{\delta} * H
\end{array}\right)(t) & =\int_{\mathbb{R}} \varphi^{\delta}(t-\eta)\left(\begin{array}{c}
E(\eta) \\
H(\eta)
\end{array}\right) d \eta \\
& =\int_{\mathbb{R}}\left[\int_{\mathbb{R}} \varphi^{\delta}(t-\eta) e^{-i(\eta-T) \lambda} d \eta\right] d \mathcal{P}_{\lambda}\left(\begin{array}{c}
y \\
0
\end{array}\right) \\
& =\int_{\mathbb{R}} e^{-i(t-T) \lambda} \rho^{\delta}(\lambda) d \mathcal{P}_{\lambda}\left(\begin{array}{l}
y \\
0
\end{array}\right) \\
& =e^{(t-T) \mathcal{M}} \rho^{\delta}(i \mathcal{M})\left(\begin{array}{l}
y \\
0
\end{array}\right)=e^{(t-T) \mathcal{M}} G^{\delta}\left(\begin{array}{l}
y \\
0
\end{array}\right)=\left(\begin{array}{l}
E^{\delta} \\
H^{\delta}
\end{array}\right)(t) .
\end{aligned}
$$


For the solution $\{E, H\}$ we have

$$
E(\cdot, T-s)=E(\cdot, T+s), \quad H(\cdot, T-s)=-H(\cdot, T+s) .
$$

Indeed, the formulas $S^{-1}=S, S \mathcal{M} S=-\mathcal{M}$ imply that

$$
e^{-s \mathcal{M}}=e^{s \cdot S \mathcal{M} S}=S e^{s \mathcal{M}} S .
$$

Now we can apply (10.3),

$$
\left(\begin{array}{l}
E(T-s) \\
H(T-s)
\end{array}\right)=e^{-s \mathcal{M}}\left(\begin{array}{l}
y \\
0
\end{array}\right)=S e^{s \mathcal{M}} S\left(\begin{array}{l}
y \\
0
\end{array}\right)=S e^{s \mathcal{M}}\left(\begin{array}{l}
y \\
0
\end{array}\right)=S\left(\begin{array}{l}
E(T+s) \\
H(T+s)
\end{array}\right),
$$

to obtain (10.14).

Under our assumptions on $f$ and $\delta$, we have $f^{\delta} \in \mathcal{F}_{0}^{T}$. By (10.4),

$$
\left(W^{T} f^{\delta}, y\right)_{J_{\theta}}=-\int_{0}^{T} d t \int_{\Gamma} d \Gamma_{x}\left\langle f^{\delta}(x, t), N H(x, t)\right\rangle .
$$

Since the inner integral is defined for all $t \in \mathbb{R}$ and is an even function with respect to $T$ (because $f^{\delta}$ and $H$ are odd) vanishing outside of the interval $(0,2 T)$, we can write

$$
\begin{aligned}
-\int_{0}^{T} d t \int_{\Gamma} d \Gamma_{x}\left\langle f^{\delta}(x, t), N H(x, t)\right\rangle & =-\frac{1}{2} \int_{\mathbb{R}} d t \int_{\Gamma} d \Gamma_{x}\left\langle f^{\delta}(x, t), N H(x, t)\right\rangle \\
& =-\frac{1}{2} \int_{\mathbb{R}} d t \int_{\Gamma} d \Gamma_{x}\left\langle\tilde{f}(x, t),\left(\varphi^{\delta} * N H\right)(x, t)\right\rangle
\end{aligned}
$$

$(\tilde{f}$ was defined in (10.10) $)$. By (10.13), for this expression we have

$$
-\frac{1}{2} \int_{\mathbb{R}} d t \int_{\Gamma} d \Gamma_{x}\left\langle\tilde{f}(x, t), N H^{\delta}(x, t)\right\rangle=-\int_{0}^{T} d t \int_{\Gamma} d \Gamma_{x}\left\langle f(x, t), N H^{\delta}(x, t)\right\rangle .
$$

By (10.4), this is equal to $\left(W^{T} f, G_{e}^{\delta} y\right)_{J_{\theta}}=\left(G_{e}^{\delta} W^{T} f, y\right)_{J_{\theta}}$, which proves (10.12).

10.3. Proof of Lemma 2. Suppose $z \in J_{\nu}$ satisfies

$$
z \perp \operatorname{clos}_{J_{\nu}} \operatorname{Ran} \mathcal{R}_{e}^{T} .
$$

Let $z^{\delta}:=G_{m}^{\delta} z \in \operatorname{Dom} \mathcal{R}_{\nu}$. Suppose $f \in \mathcal{F}_{00}^{T, s}$; then for $\delta \in(0, T-s)$ we have

$$
\begin{aligned}
\left(\mathcal{R}_{\nu} z^{\delta}, W^{T} f\right)_{J_{\theta}} & =\left(z^{\delta}, \mathcal{R}_{\theta} W^{T} f\right)_{J_{\nu}}=\left(z, G_{m}^{\delta} \mathcal{R}_{\theta} W^{T} f\right)_{J_{\nu}}=\left(z, \mathcal{R}_{\theta} G_{e}^{\delta} W^{T} f\right)_{J_{\nu}} \\
& =\left(z, \mathcal{R}_{\theta} W^{T} f^{\delta}\right)_{J_{\nu}}=\left(z, \mathcal{R}_{e}^{T} W^{T} f^{\delta}\right)_{J_{\nu}}=0 .
\end{aligned}
$$

The third identity follows from (10.9), the fourth is a consequence of (10.11). The closures of the spaces $\mathcal{F}_{00}^{T, s}$ and $\mathcal{F}_{0}^{T, s}$ in the norm of $\mathcal{F}_{+}^{T}$ coincide. Together with the continuity of the map (2.6), this means that

$$
\operatorname{clos}_{J_{\varepsilon}^{T}} W^{T} \mathcal{F}_{00}^{T, s}=\operatorname{clos}_{J_{\varepsilon}^{T}} W^{T} \mathcal{F}_{0}^{T, s} .
$$

Therefore, in view of the approximate controllability (2.8), we see that the set $W^{T} \mathcal{F}_{00}^{T, s}$ is dense in $\mathcal{U}_{\varepsilon}^{s}$. Hence, the calculation (10.16) shows that

$$
\mathcal{R}_{\nu} z^{\delta} \perp \mathcal{U}_{\varepsilon}^{s}, \quad \delta \in(0, T-s) .
$$

Now, let $y \in \mathcal{W}_{\varepsilon}^{T}$, then there exists $s \in(0, T)$ such that $y \in \mathcal{U}_{\varepsilon}^{s}$, implying

$$
\left(z, \mathcal{R}_{\theta} y\right)_{J_{\nu}}=\lim _{\delta \rightarrow 0}\left(z^{\delta}, \mathcal{R}_{\theta} y\right)_{J_{\nu}}=\lim _{\delta \rightarrow 0}\left(\mathcal{R}_{\nu} z^{\delta}, y\right)_{J_{\theta}}=0
$$

(we have already mentioned that the sequence $G^{\delta}$ converges strongly to the identity as $\delta \rightarrow 0$, whence $z^{\delta} \rightarrow z$ ). Since $y$ is arbitrary, we have

$$
z \perp \cos _{J_{\nu}} \mathcal{R}_{\theta} \mathcal{W}_{\varepsilon}^{T} \text {. }
$$


Since this relation is valid for any $z$ satisfying (10.15), we conclude that

$$
\operatorname{clos}_{J_{\nu}} \operatorname{Ran} \mathcal{R}_{e}^{T}=\operatorname{clos}_{J_{\nu}} \mathcal{R}_{\theta} \mathcal{W}_{\varepsilon}^{T} .
$$

The sets Dom $\mathcal{R}_{e}^{T}$ and $\mathcal{W}_{\varepsilon}^{T}$ are included in the space $\mathcal{U}_{\varepsilon}^{T}$, which is orthogonal to $\operatorname{Ker} \mathcal{R}_{\theta}$ (see (10.2)). From the results of Subsection 8.5 in [9], it follows that the restriction of the operator $\mathcal{R}_{\theta}$ to the orthogonal complement of its kernel has bounded inverse. Combining this with (10.17), we see that the closure $\mathcal{R}_{e}^{T}$ coincides with the closure of the restriction of $\mathcal{R}_{\theta}$ to $\mathcal{W}_{\varepsilon}^{T}$, which proves Lemma 2 .

\section{REFERENCES}

[1] M. I. Belishev, V. M. Isakov, L. N. Pestov, and V. A. Sharafutdinov, On the reconstruction of a metric from extremal electromagnetic measurement, Dokl. Akad. Nauk 372 (2000), no. 3, 298-300. (Russian) MR.1777953 (2001j:35267)

[2] M. I. Belishev and A. K. Glasman, Dynamical inverse problem for the Maxwell system: recovering the velocity in the regular zone (the BC-method), Algebra i Analiz 12 (2000), no. 2, 131-187; English transl., St. Petersburg Math. J. 12 (2001), no. 2, 279-316. MR1768140(2001i:35282)

[3] M. I. Belishev and A. S. Blagoveshchenskiı̌, Dynamical inverse problems for the wave theory, S.Peterburg. Gos. Univ., St. Petersburg, 1999. (Russian)

[4] M. I. Belishev, Recent progress in the boundary control method, Inverse Problems 23 (2007), no. 5, R1-R67. MR2353313 (2008h:93001)

[5] M. I. Belishev and V. M. Isakov, On the uniqueness of the reconstruction of the parameters of the Maxwell system from dynamic boundary data, Zap. Nauchn. Sem. S.-Peterburg. Otdel. Mat. Inst. Steklov. (POMI) 285 (2002), 15-32; English transl., J. Math. Sci. (N. Y.) 122 (2004), no. 5, 3459-3469. MR1911108 (2003e:78020)

[6] P. Ola, L. Päivärinta, and E. Somersalo, An inverse boundary value problem in electrodynamics, Duke Math. J. 70 (1993), 617-653. MR.1224101(94i:35196)

[7] Y. Kurylev, M. Lassas, and E. Somersalo, Maxwell's equations with a polarization independent wave velocity: direct and inverse problems, J. Math. Pures Appl. (9) 86 (2006), no. 3, $237-270$. MR.2257731 (2007g:35245)

[8] M. N. Demchenko, On the partially isometric transformation of solenoidal vector fields, Zap. Nauchn. Sem. S.-Peterburg. Otdel. Mat. Inst. Steklov. (POMI) 370 (2009), 22-43; English transl., J. Math. Sci. (N. Y.) 166 (2010), no. 1, 11-22. MR2749209 (2011k:35251)

[9] R. Leis, Initial-boundary value problems in mathematical physics, B. G. Teubner, Stuttgart, 1986. MR 0841971 (87h:35003)

[10] M. Eller, Symmetric hyperbolic systems with boundary conditions that do not satisfy the KreissSakamoto condition, Appl. Math. (Warsaw) 35 (2008), 323-333. MR2453536 (2009j:35212)

[11] M. Eller, V. Isakov, G. Nakamura, and D. Tataru, Uniqueness and stability in the Cauchy problem for Maxwell and elasticity systems, Nonlinear Partial Differential Equations and their Applications. Collège de France Seminar, Vol. XIV (Paris, 1997/1998), Stud. Math. Appl., vol. 31, North-Holland, Amsterdam, 2002, pp. 329-349. MR1936000 (2004c:35399)

[12] H. Sohr, The Navier-Stokes equations. An elementary functional analytic approach, Birkhäuser Verlag, Basel, 2001. MR.1928881 (2004b:35265)

[13] M. I. Belishev, On a unitary transformation in the space $L_{2}\left(\Omega ; \mathbb{R}^{3}\right)$ associated with the Weyl decomposition, Zap. Nauchn. Sem. S.-Peterburg. Otdel. Mat. Inst. Steklov. (POMI) 275 (2001), 25-40; English transl., J. Math. Sci. (N. Y.) 117 (2003), no. 2, 3900-3909. MR.1854498(2002k:35300)

[14] Yu. D. Burago and V. A. Zalgaller, An introduction to Riemannian geometry, Nauka, Moscow, 1994. (Russian) MR1356465(96h:53002)

[15] M. Sh. Birman and M. Z. Solomyak, $L_{2}$-theory of the Maxwell operator in arbitrary domains, Uspekhi Mat. Nauk 42 (1987), no. 6, 61-76; English transl., Russian Math. Ssurveys 42 (1987), no. 6, 75-96. MR0933995(89e:35127)

St. Petersburg Branch, Steklov Mathematical Institute, Russian Academy of Sciences, Fontanka 27, St. Petersburg 191023, Russia

E-mail address: demchenko@pdmi.ras.ru

Received 20/DEC/2010 